\title{
Melting and nonmelting of solid surfaces and nanosystems
}

\author{
U. Tartaglino ${ }^{\mathrm{a}, \mathrm{b}}$, T. Zykova-Timan ${ }^{\mathrm{a}, \mathrm{b}, *}$ F. Ercolessi ${ }^{\mathrm{c}, \mathrm{b}}$ \\ E. Tosatti ${ }^{a, b, d}$ \\ ${ }^{a}$ International School for Advanced Studies (SISSA-ISAS), via Beirut 2, 34014 \\ Trieste, Italy \\ ${ }^{\mathrm{b}}$ INFM Democritos National Simulation Center, Trieste, Italy \\ c Dipartimento di Fisica, Università di Udine, Via delle Scienze, 208, I-33100 \\ Udine, Italy \\ dinternational Center for Theoretical Physics (ICTP), Strada Costiera 11, 34014, \\ Trieste, Italy
}

\begin{abstract}
We present an extensive but concise review of our present understanding, largely based on theory and simulation work from our group, on the equilibrium behavior of solid surfaces and nanosystems close to the bulk melting point. In the first part we define phenomena, in particular surface melting and nonmelting, and review some related theoretical approaches, from heuristic theories to computer simulation. In the second part we describe the surface melting/nonmelting behavior of several different classes of solids, ranging from van der Waals crystals, to valence semiconductors, to ionic crystals and metals. In the third part, we address special cases such as strained solids, the defreezing of glass surfaces, and rotational surface melting. Next, we digress briefly to surface layering of a liquid metal, possibly leading to solid-like or hexatic two dimensional phases floating on the liquid. In the final part, the relationship of surface melting to the premelting of nanoclusters and nanowires is reviewed.
\end{abstract}

Key words: Surface melting; Surface phase transitions; Surface thermodynamics; Wetting; Molecular dynamics simulation.

Pacs: 61.30Hn; 64.70Dv; 68.08.-p;68.35.Md; 61.46.+w

\footnotetext{
* Corresponding author.

Email address: tzykova@sissa.it (T. Zykova-Timan).
}

Preprint submitted to Physics Reports $\quad 6$ March 2018 


\section{Contents}

1 Introduction: surface melting/nonmelting 5

$\begin{array}{lll}2 \text { Theorv: brief review } & 7\end{array}$

$\begin{array}{lll}2.1 & \text { Heuristics: solid surface instability } & 7\end{array}$

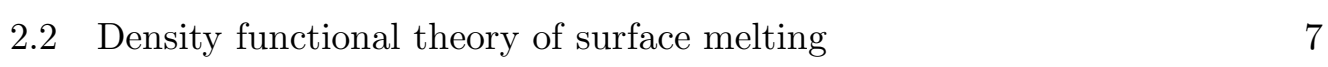

2.3 Lattice mean field theorv of surface melting (Travanov-Tosatti). and bevond 9

$\begin{array}{lll}2.4 \text { Simulations: Molecular Dvnamics } & 10\end{array}$

$3 \quad$ Surface melting and related phenomena in real solids $\quad 11$

3.1 van der Waals solids: interplav of roughening. preroughening, and melting 11

$\begin{array}{lll}3.2 & \text { Ionic insulators } & 12\end{array}$

$\begin{array}{lll}3.3 & \text { Valence semiconductors and semimetals } & 13\end{array}$

$\begin{array}{lll}3.4 & \text { Metals } & 14\end{array}$

\begin{tabular}{|ll}
$4 \quad$ Special cases & 17
\end{tabular}

$\begin{array}{lll}4.1 & \text { Strained solids } & 17\end{array}$

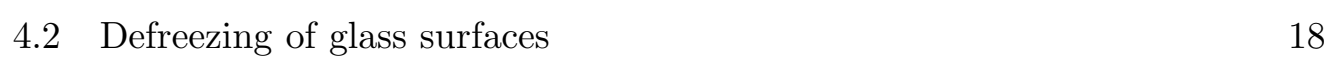

$\begin{array}{lll}4.3 & \text { Rotational surface melting in molecular solids } & 19\end{array}$

\begin{tabular}{|ll}
\hline Liquid metal surfaces & 20
\end{tabular}

$6 \quad$ Premelting of nanoclusters and nanowires 22

\begin{tabular}{|ll}
\hline & Summarv \\
\hline
\end{tabular}

\begin{tabular}{ll}
\hline References & 24
\end{tabular} 


\section{List of Figures}

$1 \quad$ Melting of a pure and homogeneous material... 28

$2 \quad$ Surface melting of $\mathrm{Pb}(110) \ldots$

$3 \quad$ Partial wetting... 30

$4 \quad$ Density functional theory of surface melting... 31

$5 \quad$ Surface melting of Lennard-Jones(110)... 32

$6 \quad$ Tvpical solid-liquid-vapor densitv profiles... 33

$7 \quad$ Reentrant lavering during the deposition... 34

$8 \quad$ Top view of the $\operatorname{Ar}(111) \ldots$

$9 \quad$ Argon bubble in contact with the solid... 36

$10 \quad$ Time evolution of $\mathrm{NaCl}$ liquid nanodroplet... 37

11 Density profile of the liquid surface of $\mathrm{NaCl}$... 38

12 Schematic Ga phase diagram... 38

$13 \quad$ Averaged atom density profile for... 39

14 Svstem with a solid branch and a liquid one... 40

15 Non-melting induced faceting trajectories... 41

16 Non-melting induced faceting of $\mathrm{Pb}(111)$ vicinals... 42

17 Evolution of Al liquid drop... 43

$18 \quad$ Simulated $\mathrm{Al}(110)$ surfaces under different strain... 44

19 Phase diagram in presence of in-plane strain... 45

20 Density profile of the liquid surfaces of $\mathrm{Pb}$ and $\mathrm{Al}$... 46

21 Densitv profile of the liquid surfaces of $\mathrm{Ar}$ and $\mathrm{NaCl}$... 46

22 Nearly hexatic top laver of Au... 47

$23 \quad$ Melting point temperature of gold particles... 48 
$24 \quad$ Melting temperature as a function of size... 49

25 Aus70: $T$ dependence of the diffusion coefficient... 50

26 Logarithm of the effective Debve-Waller factor... 51 


\section{Introduction: surface melting/nonmelting}

Melting, certainly one of the longest known phase transitions, always appeared a very special one. Although undoubtedly first order, it curiously displays only half the hysteresis cycle one would expect. In a regular first order phase transition, the free energies of two phases cross, while both remain locally stable beyond the crossing. As a result, it is generally possible to undercool the high temperature phase, and to overheat the low temperature phase. Periodic heating and cooling through a first order transition will thus generate a full hysteresis cycle.

In melting, this is only half true. It is generally possible and easy to supercool a liquid: a glass of water may not freeze at all during an icy night, only to do that suddenly when disturbed the next morning. On the contrary, it is surprisingly difficult and often impossible to overheat a free solid. The reason for that is that a free solid has surfaces; and when the melting temperature is approached, melting of the bulk crystal is ready to begin from there. Supercooling of the liquid is allowed by the absence of a solid germ; overheating of the solid is prevented by that omnipresent germ, a wet surface. This observation provides perhaps the first macroscopic hint that solid surfaces might be wet already somewhat below the melting point. The starting motivation for a microscopic study of surface melting begins right there.

Consider a semi-infinite homogeneous solid, e.g., a crystal with a free surface, in thermodynamic equilibrium at temperature $T$ and pressure $P$ with its own vapor (Fig. 1). As $T$ and $P$ are raised to approach melting at the bulk triple point $\left(T_{m}, P_{m}\right)$, where solid, gas and liquid coexist, the solid-vapor interface will generally wet itself with an atomically thin liquid film, whose thickness $\ell(T) \rightarrow \infty$ when $T \rightarrow T_{m}$. This is called surface melting (SM). A rather famous microscopic characterization of $\mathrm{SM}$ is that of $\mathrm{Pb}(110)$, first reported by Frenken and van der Veen[1]. Medium energy ion scattering data reveal (Fig. 2) the presence at the solid-vapor interface of a disordered (liquid) film whose thickness $\ell(T)$ grows without limit as $T \rightarrow T_{m}$. A number of case studies are now known in the literature, and SM turns out to be the common behavior for many surfaces. It is however not general, and many surfaces are known that do not melt in the same sense, but remain solid and crystalline all the way up to $T_{m}$. Not surprisingly, this behavior has been called surface nonmelting (NM) [2]. Slightly more surprisingly, SM and NM can both occur for the same substance, of course on two different crystallographic faces. For

example, $\mathrm{NM}$ was demonstrated for $\mathrm{Pb}(111)$ [3], while by contrast SM prevails on $\mathrm{Pb}(110)[1]$.

In the language of wetting, SM is nothing else than triple point wetting[4], namely complete wetting of the solid-vapor interface by the liquid, in other 
words wetting of the solid by its own melt. By contrast, NM corresponds to partial wetting, schematized in Fig. 3. In this case, there is a clear connection between the surface thermodynamic parameters and the angles of partial wetting [5] as will be clarified further below. Thermodynamically, NM will take place whenever the solid surface (solid-vapor interface) is unable to lower its free energy by turning into a sequence of two separate solid-liquid plus liquid-vapor interfaces, namely when

$$
\gamma_{\mathrm{SV}}<\gamma_{\mathrm{SL}}+\gamma_{\mathrm{LV}}
$$

where the $\gamma$ 's denote the free energies of the three solid-vapor (SV), solid-liquid (SL), and liquid-vapor (LV) interfaces [7]. Conversely, when the inequality (1) does not hold, SM will ensue instead of NM. As Fig. 2 shows, in SM the liquid film, although technically of divergent thickness at $T_{m}$, remains atomically thin until fractions of a degree below $T_{m}$. Interactions propagate readily across such a thin liquid film. The solid-liquid and the liquid-vapor interfaces "feel" their mutual presence, so much that they actually merge when $\ell(T)$ is small enough. For a generic temperature $T<T_{m}$ the free energy change caused by melting a solid film into a liquid one of thickness $\ell$ can be written

$$
\Delta G / A=\left[\left(\gamma_{\mathrm{SL}}+\gamma_{\mathrm{LV}}\right)-\gamma_{\mathrm{SV}}\right]+\ell L \rho_{l}\left(T_{m}-T\right)+V(\ell)
$$

where $A$ is the surface area, $L$ is the latent heat of melting per unit mass, $\rho_{l}$ is the density of the liquid, and $V(\ell)$ represents a phenomenological "interaction" free energy term between the liquid-vapor and the solid-liquid interfaces. As defined, the interaction $V(\ell)$ vanishes for $\ell \rightarrow \infty$, and tends to $V(0)=\gamma_{\mathrm{SV}}-$ $\left(\gamma_{\mathrm{SL}}+\gamma_{\mathrm{LV}}\right)$ when $\ell \rightarrow 0$, when the dry solid surface free energy is recovered. Eq. (1) amounts to say that in SM the interface interaction $V(\ell)$ is globally repulsive, whereas in NM it is attractive. A useful mnemonic is that SL-LV repulsion causes the liquid film to expand (SM), while SL-LV attraction causes the liquid film to collapse and disappear (NM).

The microscopic mechanism behind interface attraction or repulsion varies, and may be eventually related to such different physical causes as layering order [6], van der Waals forces [8], or molecular order [9]. We shall provide specific cases further below. 


\section{Theory; brief review}

\subsection{Heuristics: solid surface instability}

Early crude but appealing reasoning leading to SM has been based on heuristics. Bulk melting usually correlates well with the so called Lindemann melting criterion: when the r.m.s. thermal vibration amplitude of the solid reaches some $14 \%$ of the nearest-neighbor distance [10], solids generally melt. Atoms at surfaces are less coordinated and generally shakier than in bulk, and their vibration amplitudes are correspondingly larger. Surfaces will thus reach the Lindemann instability vibration amplitude at a lower temperature than the bulk. A model embodying the bulk Lindemann criterion is the mechanical thermal instability model [11]. An infinite solid will, if prevented from melting, become mechanically unstable at a sufficiently high temperature. Although the ideal mechanical instability temperature of a solid is different, and of course somewhat higher than the true melting temperature $T_{m}$ (where the free energy crossing of solid and liquid phases takes place), it can nonetheless heuristically be taken as a qualitative indicator of the tendency of the solid to melt. Adopting that line, one can ask whether the stability of the surface will not cease before that of the bulk. In a model semi-infinite solid it is found indeed that the mechanical instability of the first surface layer occurs at a temperature which is only $73 \%$ that of the bulk [12]. This suggests - correctly - that surfaces should begin to soften and melt at about $3 / 4$ the bulk melting temperature. That had been long known and was noted by Tammann and Stranski $[13,14,15]$. Self-consistent surface phonon calculations [16] and also experiments [17] later revealed a very pronounced anharmonic outward expansion of the first surface layer relative to that of the bulk. Although it is not really possible to gener-

alize, this is undoubtedly one of the qualitative elements heralding a stronger tendency of a surface to become unstable before the bulk.

\subsection{Density functional theory of surface melting}

The simplest proper theory of SM is based on optimizing the order parameter profile, in particular the density profile $\rho(\mathbf{x})$ of a solid-vapor interface in a system with short range interactions. Assuming a bulk grand potential density $\omega=\omega(\rho)$, the solid vapor coexistence near the triple point as in Fig. 1 implies two equivalent solid and vapor minima of the grand potential per unit volume $\omega(\rho)$ plus one additional secondary liquid minimum with a higher grand potential density. The total grand potential of the inhomogeneous system can 
be written

$$
\Omega=\iiint d^{3} x\left[\omega(\rho(\mathbf{x}))+\frac{J}{2}|\nabla \rho|^{2}\right]
$$

In this Ginzburg-Landau (or Cahn-Hilliard) type phenomenological free energy form (acceptable for a system with short range forces) the first term represents the thermodynamic potential density for a uniform system, while the gradient term signifies the extra cost caused by any spatial change in the order parameter. For a solid-vapor interface [18] we may assume $\rho=\rho(z)$ and seek to minimize $\Omega$ (per unit area):

$$
\Omega / A=\int_{-\infty}^{+\infty} d z\left[\omega(\rho(z))+\frac{J}{2}\left(\frac{d \rho}{d z}\right)^{2}\right]=\min
$$

with the constraints $\rho(-\infty)=\rho_{s}$ and $\rho(+\infty)=\rho_{v}$, where $\rho_{s}$ and $\rho_{v}$ are solid and vapor densities respectively. Amusingly, this minimization problem is formally identical to the minimum action problem for the one dimensional lagrangian motion of a classical point object of coordinate $\rho$, mass $J$, as a function of time $z$, in a potential $-\omega(\rho)$. Starting at time $z=-\infty$ with zero kinetic energy and with a small $\rho=\rho_{v}$ from a first hilltop of height $-\omega\left(\rho_{v}\right)$ (the vapor phase), the point moves "downhill" eventually reaching at time $z=+\infty$ the last, and exactly equivalent, hilltop $-\omega\left(\rho_{s}\right)=-\omega\left(\rho_{v}\right)$ (the solid phase) (Fig. 4).

At temperatures well below the melting point, there is no liquid phase (not even metastable), meaning that there exists no liquid-like local minimum in $\omega(\rho)$. Thus $-\omega(\rho)$ has no other maxima than the vapor and solid hilltops, and the resulting solid-vapor interface is unsplit and featureless. Close to $T_{m}$ however $-\omega\left(\rho_{v}\right)$ develops a secondary maximum - a lower hilltop, as it wereat an intermediate $\rho=\rho_{l}$. En route from the vapor to the solid hilltops, the point particle must negotiate this intermediate "mountain pass", where it will not stop, but will still slow down considerably. It is easy to check that if $\Delta=-\omega\left(\rho_{s}\right)-\left(-\omega\left(\rho_{l}\right)\right)$ is the height difference between main and secondary hilltops (the free energy difference between solid and liquid, that goes to zero only at the melting point), then the time $\delta z$ the point will spend near the liquid hill, and therefore with $\rho \approx \rho_{l}$ is proportional to $\log \Delta$. Thus the interface density profile is now split into two interfaces: first a vapor-liquid one, then a liquid-solid one. They are separated by a liquid film of density close to $\rho_{l}$, whose thickness $\ell=\delta z$ is logarithmically increasing as $T \rightarrow T_{m}$. And this is of course just surface melting.

The above also suggests that one might identify some temperature $T_{w}<T_{m}$ where the bulk free energy first develops the local minimum corresponding 
to the liquid phase, with the temperature $T_{w}$ where a solid surface is likely to begin wetting itself with the thinnest liquid film. It must be underscored that this simple theory only describes SM and cannot account for NM or for many other complications, upon which we shall return below. The presence of a "surface term" in Eq.(4) will additionally modify this scenario[18].

\subsection{Lattice mean field theory of surface melting (Trayanov-Tosatti), and be- yond}

The discussion above is centered on density as the sole order parameter. A solid actually differs from the liquid not just by the average atomic density $\rho_{0}$, but also by the infinite set of crystalline Fourier components of the atomic density $\rho_{\mathbf{G}}$ ( $\mathbf{G}$ denoting reciprocal lattice vectors) that are nonzero only in the solid. A better order parameter theory of SM than that outlined above should be based on a free energy expression that correctly includes these crystalline order parameters. Trayanov and Tosatti [19] built a simplified but microscopic two-order parameter lattice theory based on the average density $\rho_{0}$ plus a second "crystallinity" order parameter $c$, the latter effectively replacing the less manageable infinite set of $\rho_{\mathbf{G}}$. Through a further mean field approximation where lattice layers are assumed to behave uniformly (thus neglecting roughening fluctuations), realistic systems such as Lennard-Jones (LJ) solid surfaces can be described in this manner. Fig. 5 illustrates the surface melting of fcc LJ(110) described by this lattice theory. The approach also demonstrated how in the LJ systems the logarithmic liquid film thickness divergence close to $T_{m}$ is asymptotically replaced by a more realistic $\left(T_{m}-T\right)^{-1 / 3}$ divergence, due to the (omnipresent) van der Waals long range interatomic potential tails. Let us expand briefly on this point. Given three media S, L and V, with a finite thickness $\ell$ of L sandwiched between semi-infinite $S$ and $V$, there will generally arise a long range tail to the interaction free energy between the two interfaces

$$
V(l) \sim H / \ell^{2}
$$

due to long range dispersion forces. The so-called Hamaker constant $H$ is generally positive when the intermediate $\mathrm{L}$ phase is dielectrically less dense that $\mathrm{S}$, and more dense than $\mathrm{V}[20]$. Positive $H$ means long range repulsion between the interfaces, which favors SM. That is precisely the case for the LJ-like systems, where the liquid is less dense than the solid. The same will generally also apply to many metals and other materials. In different cases, such as valence semiconductors and semimetals, melting leads to a denser and generally metallic liquid state, and there $H<0$. Negative expansion at melting and a negative Hamaker constant occur also in other notable cases, water among them $[21,22]$. Here $H<0$ means a long range attraction between interfaces, and that implies NM. As we shall see below, the surfaces of a 
semiconductor actually appear to wet close to $T_{m}$. However the wetting is incomplete, and the melted film thickness $\ell$ remains microscopically thin, in agreement with their negative Hamaker constant.

Of course mean field theories, such as Trayanov-Tosatti above, are only an approximation to a real critical phenomenon. Mean field is usually a bad approximation sufficiently close to a critical point (in SM the critical point is $T=T_{m}$ ), where the correlation length (here signified by the liquid thickness $\ell)$ diverges. In systems with short range forces critical fluctuations may lead to nonclassical exponents. For SM, this aspect was discussed theoretically by Lipowsky and collaborators [18], and later by Chernov and Mikheev[23]. It was also addressed through atomistic simulations by Chen et al [24]. The conclusion is that although non-mean field fluctuations (mostly roughening fluctuations) actually have a large quantitative effect [24], they will not change the mean field exponent $-1 / 3$ which originates from long range forces [8].

\subsection{Simulations: Molecular Dynamics}

Atomistic simulations constitute a very important tool in the field of melting and surface melting. They can be either of Monte Carlo (MC) type, or of Molecular Dynamics (MD) type. We shall concentrate on the latter, using work done in our group for specific examples. SM simulations are generally conducted on crystal slabs, made up of a sufficiently large number of atomic solid layers, with two free surfaces, or with one free and one "frozen" (fixed atoms) surface, and periodic boundary conditions along the two directions parallel to the surfaces. The in-plane simulation cell size must be allowed or otherwise made to expand gradually as temperature increases, to guarantee that the planar stress in the solid on account of thermal expansion remains as close as possible to zero. The simulation consists of solving Newton's equations of motion

$$
M_{i} \ddot{\mathbf{R}}_{i}=-\nabla_{i} E_{\text {pot }}(\{\mathbf{R}\})
$$

for all particles of mass $M_{i}$, and coordinates $\mathbf{R}_{i}$. The basic input needed is the potential energy $E_{\text {pot }}(\{\mathbf{R}\})$ as a function of all coordinates. Depending on systems, and on the accuracy needed, the choice is between empirical interatomic potentials on one side, and first principles total energy calculations $E_{\text {pot }}(\{\mathbf{R}\})$ on the other side. Empirical two-body potentials, such as LennardJones (LJ), are quite reasonable for rare gas solids or van der Waals molecular crystals, but not for most other solids. For metals, empirical many body potentials such as the Embedded Atom Model [25], the similar glue model [26], the Finnis-Sinclair potential [27] etc., are much more suitable than two-body forces. Many-body empirical potentials were developed also for such systems 
as valence semiconductors $[28,29]$ but here the first principles simulation approach is generally much more appropriate [30]. Typical SM simulation density profile outputs are shown in Fig. 6 for a modified LJ system and for a Au metal surface respectively. Technical details and some further results for simulations of SM can be found in the review article by Di Tolla et al [31]. Simulations may in many ways replace experiment, and must be similarly regarded and understood by means of theory - not discounted as self-explanatory as it is sometimes tempting to do. Similar to experiment, they do uncover (occasionally by serendipity), novel or unexpected behavior. Such has been the case for surface nonmelting, first discovered in simulation [2] and next found experimentally [3], and several other phenomena, to be discussed further below.

\section{Surface melting and related phenomena in real solids}

\section{1 van der Waals solids: interplay of roughening, preroughening, and melt- ing}

Rare gas solids are reasonably described through a Lennard-Jones potential. As first shown by extensive MD studies by Broughton and collaborators $[32,33]$ they are expected to display surface melting on all crystalline faces. Here truly microscopic experiments are not abundant, because of technical difficulties. There is however a large body of work by the surface adsorption community, interested in understanding adsorption isotherms as a function of temperature [34].

Adsorbtion of atoms on a substrate generally takes place in a layer-by-layer mode, up to the so-called surface roughening temperature $T_{R}$, where the interface width diverges and layers cease to exist. Surface roughening-a KosterlitzThouless phase transition taking place (sometimes but not always) at a temperature $T_{R}<T_{m}$-is a totally distinguished phenomenon from surface melting [35], but it does have some relations with it. It can be argued for example that surfaces that undergo SM at $T=T_{m}$ will necessarily undergo a roughening transition at $T_{R}<T_{m}$ [36]. That is because a liquid surface is technically rough, so that capillary fluctuations cause the liquid-vapor interface width to diverge. Conversely, although there is apparently no theorem that says so, a NM surface will usually not undergo roughening below $T_{m}$. Disregarding connections with surface melting - even though they are sometimes important, as discussed below-roughening is generally discussed in purely lattice models.

There is yet another surface phase transition that may occur below melting, in fact below roughening, therefore called preroughening (PR) $[37,38,39,40]$. PR is associated with the bulk lattice planes having some stacking such as abab... 
or $a b c a b c \ldots$ where planes $a$ and $b$ can only be made to coincide through a fractional translation. At low temperatures a perfect surface, say a (100) face, will with identical probability be either $a$ or $b$ terminated. As in an Ising model, either choice will "spontaneously break" the symmetry between $a$ and $b$. At the PR temperature $T_{P R}$, a surface restores precisely the $a-b$ symmetry in an Ising-like fashion. To achieve that, the topmost atomic layer undergoes a most surprising spontaneous rearrangement, from full coverage below $T_{P R}$, (full $a$

or full $b$ ) to $\frac{1}{2}$ coverage above $T_{P R}$. The excess atoms simply migrate away, turning the top half layer into a sort of soft a-b "checkerboard". Strictly at $T=T_{P R}$ the surface becomes technically rough, only to become flat again between $T_{P R}$ and $T_{R}$. Preroughening was first discovered theoretically in lattice models [37], and received little attention by experimentalists until the equally surprising phenomenon of re-entrant layering was discovered [41,42]. Adsorbtion of Ar, Xe, Kr on a graphite substrate (Fig. 7) proceeded by full layers below $0.82 T_{m}$. This appeared to be roughening: but layering surprisingly reentered at higher temperature, this time by half layers. While that clearly called for an explanation in terms of preroughening [43] a considerable controversy arose, as independent evidence suggested that the first surface layer was in reality no longer solid, with considerable liquid-like disorder and first layer mobility in that temperature regime [44]. Moreover, reentrant layering signaled a clear first order transition, whereas PR should be second order. The resolution of this puzzle, reached through theory [45], grand canonical MC [46,47] and MD [48] simulations, proved to be that PR is linked to first (half-) layer surface melting: in essence both phenomena take place simultaneously. PR in a rigid lattice surface model would indeed only take place at higher temperatures, and the half monolayer would in that case be stabilized through a second order transition. In real off-lattice Argon however the half monolayer soft checkerboard immediately melts, with a first order transition and a large positional entropy gain over the corresponding hypothetical solid half monolayer. As a result, preroughening and first layer melting actually take place simultaneously, at a much lower temperature than either of them separately, and of course through a first order transition. Fig. 8 shows a realistic snapshot picture, obtained by MC, of the $\operatorname{Ar}(111)$ surface simulated in the reentrant layering temperature regime, showing the spontaneous formation of a roughly half-coverage, liquid like top monolayer.

\subsection{Ionic insulators}

Surfaces of ionic crystals such as $\mathrm{NaCl}$, other alkali halides, $\mathrm{MgO}$, etc, are often used as substrates for growing other materials, and their properties are therefore better known far below the melting point. Yet, some data are also available about their behavior at $T_{m}$. 
In this respect, $\mathrm{NaCl}$ appears to be one of the best studied, and can therefore be chosen as a case study. Argon bubble studies of liquid $\mathrm{NaCl}$ in contact with the solid revealed a surprising lack of complete wetting, with a large partial wetting angle of about 48 degrees (Fig. 9)[49] . Very recent MD simulations of solid $\mathrm{NaCl}(100)$ clearly demonstrates $\mathrm{NM}$ of this solid surface, also predicted to survive in a metastable state well above $T_{m}[9]$. Simulations of a droplet of melted $\mathrm{NaCl}$ brought into contact with solid $\mathrm{NaCl}(100)$ at $T=T_{m}$ (Fig. 10) clearly shows incomplete wetting, with an external partial wetting angle of $(50 \pm 5)^{\circ}$, in good agreement with the bubble experiments [50].

The microscopic reasons for the exceptional stability of the solid $\mathrm{NaCl}(100)$ surface and its unreadiness to wet itself are presently under scrutiny. They are intriguing in view of a large positive Hamaker constant - $\mathrm{NaCl}$ undergoing a $26 \%$ volume expansion at melting - which would suggest the opposite behavior, namely SM. A second element wrongly suggesting SM against NM is the structure of the liquid surface, very strongly fluctuating and totally devoid of layering (Fig. 11) unlike e.g., nonmelting metal surfaces (see below). So why does strong NM arise in alkali halides?

Preliminary useful clues come from surface thermodynamics. MD simulations permit the explicit calculation of $\gamma_{L V}, \gamma_{S L}$ and $\gamma_{S V}$ as a function of $T$ [9]. It appears that while $\gamma_{S V}$ drops very strongly in virtue of large surface relaxations and the associated growing vibrational entropy at $T \sim T_{m}$, the liquid surface is incapable of developing a competing amount of entropy, presumably because of the all-important local charge neutrality constraint, and of the related appearance of molecular order in the fluctuating liquid $\mathrm{NaCl}$ surface. This physical result is the subject of current work [96].

\subsection{Valence semiconductors and semimetals}

Valence semiconductors such as $\mathrm{Si}, \mathrm{Ge}$, GaAs and semimetals like Ga are known to turn fully metallic and denser either at high pressure or when they melt at high temperature. This is illustrated by the schematic Ga phase diagram of Fig. 12. The higher liquid density here causes the Hamaker constant to be negative [24], and this necessarily hinders complete surface melting. Reports of regular SM with unlimited growth of the liquid film at $T_{m}$ that have appeared for $\mathrm{Ga}[51]$ are incompatible with the negative Hamaker constant, and might in our view be an artifact, possibly due to strains (see below). Due to the long range attraction, regular and complete SM is excluded for all faces of these $H<0$ materials.

The clean surfaces of semiconductors, Ge(111) and $\mathrm{Si}(111)$, were thoroughly investigated for surface melting. While they were found to disorder [52] and 
simultaneously to become metallic [53] on the topmost layer at some $T<T_{m}$, there is clear evidence that the disordered film does not grow as $T_{m}$ is approached. In order to understand theoretically the top layer disordering and metallization, MD simulations are perhaps the best tool. Since electronic effects and the semiconductor-metal transition play a very important role in the melting of these systems, it seems mandatory to take recourse in this case to first-principles simulations, where forces acting on the ions are obtained by solving the full electronic structure problem. Simulations of this type, pioneered by Car and Parrinello in the mid 80s [54], are by their nature much more expensive and demanding than the empirical ones. Results for Ge(111) by Takeuchi et al [30] are shown in Fig. 13 at $T \sim T_{m}$. As signaled by the broader density profile, the topmost bilayer is indeed strongly disordered, but disorder does not propagate to the second bilayer, indicating incomplete melting. Electronically, the surface disordered film is metallic [53], as opposed to the underlying bulk, which remains semiconducting.

\subsection{Metals}

The surface melting behavior of metallic surfaces has received by comparison much more experimental attention. Metal surfaces usually possess generally positive Hamaker surfaces, and generally melt. In detail, they may exhibit either melting and nonmelting, depending on the metal and on the crystallographic orientation. A minority of close packed surfaces such as $\mathrm{Pb}(111)$ [3], $\mathrm{Al}(111)$ and $\mathrm{Al}(100)$ [55], etc. display $\mathrm{NM}$, and remain in fact smooth and dry all the way to $T_{m}$. The vast majority of metal faces consisting of all other orientations, where packing is poorer, undergo SM. In intermediate packing cases like for instance $\mathrm{Pb}(100)$ [3], the first few layers melt, but the wetting does not proceed and the liquid film growth is blocked to a finite thickness until $T_{m}$ (incomplete $\mathrm{SM}$ ). Although this incomplete melting will macroscopically appear just the same as NM-namely partial wetting with a finite wetting angle - there is a clear microscopic difference because the surface is no longer dry below $T_{m}$.

An early review of SM on metals can be found elsewhere[52]. We will limit ourselves to mention here a few high-temperature metal surface phenomena, particularly those connected with NM, which have been especially highlighted in our group.

The first is the existence in surface NM of a critical liquid nucleation thickness $\ell_{\text {crit }}$ which is finite above $T_{m}$ and only vanishes at some "surface spinodal temperature" $T_{s}>T_{m}$. This fact, first discovered in simulation [2], indicates that in NM the solid surface is in fact metastable, and protected by a nucleation barrier, between $T_{m}$ and $T_{s}$. An immediate consequence is that NM 
solid surfaces can be overheated, of course only for a short time, up to ati most $T_{s}>T_{m}$. For the (111) surfaces of $\mathrm{Pb}, \mathrm{Al}$ and $\mathrm{Au}$ the calculated amount of maximum theoretical surface overheating $T_{s}-T_{m}$ is $120 \mathrm{~K}, 150 \mathrm{~K}$, and $150 \mathrm{~K}$ respectively. This possibility was demonstrated experimentally by laser heating techniques [56], and also in small $\mathrm{Pb}$ clusters [57].

Another phenomenon discovered theoretically is non-melting induced surface faceting. Consider a general crystal surface, whose orientation is close to, but not exactly coincident with, a flat NM face. Such a "vicinal surface" will consist of a sequence of flat terraces separated by surface steps. At low temperature the steps will usually repel mutually and form an ordered array, so that the solid vicinal surface is stable. As $T_{m}$ is approached, the steps suddenly coalesce in bunches giving way to much larger flat terraces, separated by very inclined facets where the steps have bunched up. The inclined facets with high step density actually melt, while the step-free flat facets remain dry. This nonmelting induced surface faceting was first predicted thermodynamically [58], as seen on the free energy sketch (Fig. 14), demonstrated by MD simulation (Fig. 15) [59], and eventually observed experimentally (Fig. 16) [60] . It is most likely the reason why e.g., beautifully perfect flat faces can be generated in gold, by simple heating close to the melting point. $\mathrm{Au}(111)$ being a nonmelting face, it will at high temperature cause all steps and imperfections to bunch up in some place, sweeping itself clean and flat.

A third interesting point is the microscopic investigation and clarification of the relationship between solid surface NM and its partial wetting by a drop of melt. While macroscopic partial wetting with a nonzero contact angle of the liquid with its own solid is not commonly reported for metals, it is very clearly found by simulation. Fig. 17 shows the contrasting fate of a simulated Al liquid nanodroplet at $T=T_{m}$ when approaching alternatively a $\mathrm{SM} \mathrm{Al}(110)$ surface, or a $\mathrm{NM} \mathrm{Al}(111)$ surface. In the first case, the droplet spreads rapidly and disappears. In the second it settles down to a metastable state, forming the nanoscopic equivalent of a partial wetting angle $\theta_{L V}=(22 \pm 3)^{\circ}$.

There is in fact, in a simple model, a direct relationship between $\theta_{L V}$ and the overheating $O=T_{s}-T_{m}$ of a NM surface, namely

$$
\frac{T_{s}}{T_{m}}=1+\frac{2 \gamma_{L V}}{L \rho_{l} \xi} \sin ^{2} \frac{\theta_{L V}}{2}
$$

where $L$ is the latent heat of melting per unit mass, $\rho_{l}$ is the density of the liquid, $\xi$ is the correlation length in the liquid. The background of this formula is Eq. (2) with a short range interface interaction

$$
V(\ell)=V(0) \exp -(\ell / \xi)
$$


plus the appropriate Young equation $[5,58]$

$$
\gamma_{S V}=\gamma_{L V} \cos \theta_{L V}+\gamma_{S L} \cos \theta_{S L}
$$

where moreover $\theta_{S L} \sim 0$ at $T=T_{m}$. Based on Eq. (7) the calculated ideal overheating temperatures of $\mathrm{Pb}(111), \mathrm{Al}(111)$ and $\mathrm{Au}(111)$ given earlier above predict partial self-wetting angles $\theta_{L V}$ of $(16 \pm 1)^{\circ},(18 \pm 2)^{\circ},(33 \pm 2)^{\circ}$ respectively. By comparison $\theta_{L V}$ is found to be $(14 \pm 1)^{\circ}[60]$ in the experiment for $\mathrm{Pb}(111)$, and $(22 \pm 2)^{\circ}$ in the simulation for $\mathrm{Al}(111)$ (see above).

In addition to the above thermodynamics, there is a simple microscopic understanding of the physics that leads to NM of these close packed metal surfaces $[5,31]$. It has to do with the intrinsic atomic structure of the two SL and LV interfaces involved. A series of $z$-resolved in-plane averaged density profiles of the SL and LV interfaces as displayed by MD simulations of suitably prepared liquid films on $\mathrm{Au}(111), \mathrm{Au}(110)$, and $\mathrm{LJ}(111)$ near their respective $T=T_{m}$ is shown in Fig. 6. A well defined damped density oscillation is seen to propagate from the solid into the liquid, carrying approximately the solid interplanar distance as a wavelength. This wavelength of course depends on the crystallographic direction; in the present example it is large for (111) but small for (110). A second damped density oscillation starts at the liquid surface, carrying inward a generally different wavelength, determined this time by the main peak in the liquid structure factor $S(k)$. This second oscillation, essentially non-existent in the LJ liquid, is generally strong in a metal (see discussion further below). Being a property of the liquid, the surface layering oscillation of the surface melted film is obviously face independent.

When, as in $\mathrm{Au}(110)$, the solid and liquid oscillations facing one another possess wavelengths that are out of tune, then their superposition is unfavorable and causes interface repulsion, eventually leading to SM. This is what happens for $\mathrm{Au}(110)$. When instead, as in $\mathrm{Au}(111)$, the two oscillations are close to being perfectly tuned, their superposition is favorable and causes interface attraction, leading to NM. Finally, the practical absence of a layering oscillation in the LJ liquid surface indicates indifference of the two interfaces. They nonetheless eventually interact via the positive Hamaker constant [Eq. (5)], and therefore SM ensues in that case too. 


\section{Special cases}

\subsection{Strained solids}

In an infinite bulk solid, a shift $\Delta P$ of external pressure provokes a shift of the bulk melting temperature by an amount $\Delta P\left(v_{l}-v_{s}\right) /\left(s_{l}-s_{s}\right)$, where $v_{l}$ and $v_{s}$ are the molar volumes of the liquid and the solid phase respectively, and $s_{l}$ and $s_{s}$ are the corresponding molar entropies. Thus an increase of the external pressure generally increases the melting temperature. Contrary to this, a uniaxial strain always works the other way around and favors melting, irrespective of the sign of strain. Uniaxial compression or stretching increases the elastic energy of the solid (so long as the solid can sustain the corresponding stress without relevant plastic deformation). On the other hand the liquid does not support shear, and all elastic energy is released by flow. The bulk melting temperature of a strained solid is thus lowered by an amount which is quadratic in the anisotropic strain. This effect has been experimentally observed in He crystals [61]. In metals it has been studied theoretically through simple thermodynamics, and accessed directly by molecular dynamics simulations for aluminum $[62,63]$. This is therefore really a bulk effect though it will readily show up at surfaces, as shown by the simulation results of Fig. 18. Due to the strain-induced lowering of the bulk $T_{m}$ the liquid film thickness at fixed temperature on $\mathrm{Al}(110)$ increases strongly and quadratically with strain, due simply to strain induced lowering of $T_{m}$. A corollary of some practical importance is that in any study of surface melting the strain conditions must be severely controlled. The Al test case indicates that a relatively small strain of $10^{-3}$ implies a shift of $T_{m}$ of 0.03 degrees, or $0.003 \%$. As such a degree of precision is sometimes approached in surface melting studies, the error introduced by strains can be significant. Since strain conditions are seldom specified explicitly, it seems possible and in some cases likely that some of the asymptotic SM data in the literature might need re-checking against that source of error.

Besides this bulk effect, there are more interesting strain-related surface effects. The first is the so-called Asaro-Tiller-Grinfeld instability, an experimentally well established effect; the second, is a strain-induced prewetting and eventual NM of an initially SM surface, an effect so far only hypothetical, but observed in MD simulation.

The Asaro-Tiller-Grinfeld instability $[64,65]$ is a periodic wavy modulation of the interface between the liquid and the strained solid, once the in-plane strain exceeds a critical threshold. It is connected with the possibility, permitted by a wavy interface only, of the solid "promontories" sticking out into the liquid to relax, reducing their strain and the associated elastic energy. When the bulk strain magnitude is large, the local surface strain release becomes large 
enough, and pays for the free energy cost associated with the extra interface area due to the waviness. This effect has been observed at the He solid-liquid interface by the Ecole Normale group [61].

Strain induced prewetting can instead occur because a SV interface will generally possess, in full equilibrium, a nonvanishing surface stress. For instance most metal surfaces are known to possess a large tensile surface stress, signifying that the topmost layers would really like to contract relative the bulk, whose lattice spacing is of course fixed [66]. When an in-plane bulk strain is applied, surface stress will work against the strain, or along with the strain, and the work reversibly done or released will increase or decrease respectively the overall SV surface free energy $\gamma_{S V}$. As seen in $\mathrm{Eq}(1)$ and (2) a straininduced increase of $\gamma_{S V}$ will further encourage SM, but a decrease will oppose it. In the latter case it can be shown (Fig. 19) in the very simple model of ref. [62] that strain will cause ordinary, continuous SM to be replaced by a prewetting transition: as $T$ grows surface melting ceases to be continuous, and develops a sudden jump in the liquid film thickness $\ell$ from zero to a finite value, from where it then grows continuously and diverges at $T \rightarrow T_{m}$ as in regular SM. This strain-induced prewetting transition has recently been confirmed by simulations [63], but has yet to be experimentally pursued.

\subsection{Defreezing of glass surfaces}

We dealt thus far with surfaces that are not just solid, but also crystalline. Many solids are however not crystalline, but amorphous or glassy. A glass does not break translational invariance the way a crystal does. Still, it breaks ergodicity in exactly the same manner as the crystal. Due to freezing and to the ensuing dynamical arrest, at the bulk glass temperature $T_{g}$ a glass, similar to a crystal, ceases to explore the full configurational phase space in the extensive way a liquid does, and acquires rigidity [67], the hallmark of broken positional ergodicity.

Let us consider a semi-infinite glass with a free surface, and let $T$ increase: will the glass surface defreeze at the same temperature $T_{g}$ as the bulk glass? In principle there is no simple answer to that, because the transition to the glassy state is a matter of dynamics, and not of thermodynamics. One can nonetheless build a simple "enthalpy functional approach", different in spirit but relatively parallel in reasoning, to the density functional theory of SM of Sec. 2. This approach is in turn based on a recent thermodynamic formulation of the glass transition, where this transition is in fact second order $[68,69]$.

The enthalpy functional analog of Eq. 4 [70] which represents the surface version of the thermodynamical glass theory indeed predicts a surface defreezing 
beginning at a lower temperature than the bulk $T_{g}$, with a penetration depth that also grows logarithmically as $T \rightarrow T_{g}$, superficially similar to $\mathrm{SM}$ in crystal surfaces. At a closer look, surface glass defreezing is in reality a much weaker affair than crystal SM is. To illustrate this, one can note a very substantial difference in the amount of total surface diffusivity, given by the integral of surface diffusivity over depth. This integral, measuring the amount of real flowing liquid at the surface, is divergent at $T_{m}$ for a SM crystal surface, but remains finite up to $T_{g}$ at a glass surface.

Glasses may be expected to flow very, very slowly. If the flow takes place in the bulk, a piece of glass with a given initial shape will generally change its shape in a well defined manner [71]. The expected shape change will be totally different if the flow takes place at the surface instead of the bulk [72]. Unlike bulk flow, surface flow should wipe out sharp corners and edges, rounding off a finite but increasing length away from the corner as a function of time. It would be very interesting if these predictions, so far purely theoretical, could be explored experimentally, e.g., by studying shape changes of glasses by centrifuge experiments.

\subsection{Rotational surface melting in molecular solids}

We have been concerned with surfaces consisting implicitly of either atomic elements, or of small molecules such as $\mathrm{NaCl}$ merging together to form the solid. Let us consider by contrast molecular crystals made up of molecules that do not merge when crystallizing, and behave so to speak as nanosized rigid bodies, both in the crystal and in the liquid phase. In that case thermal disordering will concern, besides the positional degrees of freedom of the molecular centers of mass, also the rotational degrees of freedom. For very asymmetrical molecules, it can be expected that the two sets of degrees of freedom will be closely entangled. For nearly spherical molecules on the other hand the rotational and positional phenomena will involve very different energies and therefore different temperatures, the rotational well below the positional. In that situation one can have rotational melting, a first order phase transition transforming a proper crystal into a "plastic crystal" (somewhat of a misnomer) where the molecular centers of mass are still positionally crystalline, but where the rotational coordinates are thermally disordered, or melted. A particularly popular example of this behavior is provided by $C_{60}$ fullerite, where rotational melting is known to take place at $T_{\text {rot }} \sim 250-260 \mathrm{~K}[73]$, whereas there is in $C_{60}$ no positional melting, and essentially no liquid phase.

Consider the surface of such a molecular crystal and let $T$ increase towards $T_{\text {rot }}$. Will there be a rotational equivalent to surface melting, the first few layers thermally disordered and the bulk still rotationally ordered? The answer is in 
principle yes; and again, simple free energy functionals can be resorted to in order to explore that scenario [74]. Here, perhaps the most interesting aspect here, (and certainly the most relevant to $C_{60}$, where an abrupt surface phase transition was detected some 30 degrees below $T_{\text {rot }}$ [75]) appears to be the earliest stages of surface rotational disordering.

There is a crucial difference between rotational and positional melting. Even in a rotationally melted plastic crystal, a molecule is still surrounded by a lattice, its cage exerting onto the molecule a sort of crystal field potential. In the ordinary liquid instead there is no remaining lattice at all. In the molecular solid, the crystal field potential felt by a first layer surface molecule is very different from the bulk one. The early stages of surface disordering and their precise nature will be vastly determined by that difference. Calculations show that at the surface of $C_{60}$ the crystal field actually frustrates bulk order; moreover it does so differently for different surface molecules. This gives in fact rise to a first layer thermal disordering process that proceeds in two stages, the more frustrated molecules disordering at a lower temperature, the less frustrated at higher temperature (but still below the bulk $T_{\text {rot }}$ ) [76].

Similar scenarios could be expected to take place for other molecular crystals, where surface studies are however not yet abundant so far.

\section{$5 \quad$ Liquid metal surfaces}

Our focus throughout has been on solid surfaces, touching only incidentally upon the physics of liquid surfaces, whenever that was important for surface melting. Yet, liquid surfaces have so to speak a life of their own. Liquid metal surfaces were long known to be interesting and structured. As revealed by e.g., X-ray reflectivity experiments $[77,78]$, and as discussed long ago by chemical physicists such as Rice [79], the density profile of liquid metal surfaces shows a tendency to layering. Figs. 20 and 21 show as an example the density profiles obtained by simulation for the $\mathrm{Pb}, \mathrm{Al}$, LJ and $\mathrm{NaCl}$ liquid surfaces at their respective $T_{m}$.

Layering is in principle easy to understand, and simply represents the density response of the liquid to vacuum. Like all response functions, it is intimately related to the internal structure of the liquid, very thoroughly described in standard textbooks [80]. In short, if $g(r)$ is the liquid pair correlation function, it will exhibit a main (damped) oscillation with some typical liquid interparticle spacing $\lambda$. Its Fourier transform is the liquid static structure factor $S(k)$, which is correspondingly peaked around $k_{0}=2 \pi / \lambda$. When an external perturbation, say a $\delta$-function point-like repulsion, is inserted, the liquid density will locally drop, and will recover away from the point with a damped oscillation 
dictated by $S(k)$, and with a wavelength close to $\lambda$. The surface represents another perturbation of that type (now extended) for the liquid metal, and layering is the result. Of course, the perturbation represented by vacuum is small only deep below the liquid surface, but is very large in the outermost layer. Here the precise form of layering will generally take a different form from that suggested by perturbative considerations.

The metal atoms in the outermost surface layer are roughly speaking confined in two dimensions. Moreover, in some cases - like in the heavy noble metals that are inclined to surface reconstructions - the packing of surface atoms will tend to be closer and tighter than the corresponding bulk atoms. The question therefore arises whether the outer surface of a liquid metal could in some cases go as far as crystallizing in two dimensions (2D). Such a crystallization would represent a case of "surface freezing" - the opposite of surface melting - a phenomenon demonstrated and characterized in alkanes [81,82]. The additional interest of pursuing this in elemental liquid metals would be the possibility to use this for a particularly delicate test of the theories of two-dimensional freezing [83].

In two dimensions, freezing takes place in two steps. Disclination pairs should bind into dislocations with a first transition, implying the onset of a "hexatic phase" with power law orientational order. Upon further cooling, a second transition binds dislocation pairs, with the onset of power law positional order. These phenomena have been well documented in the melting of a $2 \mathrm{D}$ colloidal crystal [84]. A liquid metal surface might be able to exhibit, either above $T_{m}$ or in the supercooled state, a hexatic phase. Floating on top of a three dimensional liquid, the layered, nearly 2D solid surface would be able to exchange freely atoms with it, and disclinations/dislocations could readily form and dissolve avoiding all the delicate kinetic problems presented by strict 2 D systems.

Celestini et al [85] carried out extensive MD simulations of the liquid Au surface as a function of temperature particularly in the supercooling regime. As Fig. 22 shows, an increasingly strong layering is indeed expected, and the top layer strongly resembles a $2 \mathrm{D}$ close packed lattice with a large number of disclinations. Upon supercooling, disclinations rarefy and the system approaches a hexatic transition. On the brink of that transition however, bulk crystallization suddenly takes place, starting precisely from the liquid surface inwards [86].

An experimental attempt at pursuing experimentally the possible formation of $2 \mathrm{D}$ hexatics at liquid metal surfaces would seem very desirable, particularly with powerful tools such as surface X-ray reflectivity. Unfortunately, it appears that high melting point metals such as $\mathrm{Au}, \mathrm{Pt}$ and Ir, where layering and 2D crystallization tendencies are particularly strong, are technically out of reach at least with this technique [77]. 


\section{Premelting of nanoclusters and nanowires}

Nanosystems became only recently a hot subject, but their thermal behavior has long been an important one. Small clusters liquefy readily, or "premelt", well below the bulk melting temperature of the same material. Basic thermodynamics predicts phenomenologically that a cluster of radius $R$ should melt at $T_{m}(R)$

$$
T_{m}(R) \approx T_{m}\left\{1-\frac{2}{\rho_{l} L R}\left[\gamma_{S V}-\left(\gamma_{S L}+\gamma_{L V}\right)\right]\right\}
$$

This behavior was well verified experimentally in Au by classic cluster beam experiments by Buffat and Borel [87], whose results are shown in Fig. 23. A very parallel melting behavior is expected for one-dimensional extended nanosystems, such as nanowires [88]. In that case that Eq. (10) remains valid, although without the factor 2 , as has also been confirmed by simulations.

Microscopic theories as well as atomistic MD simulations of cluster melting are numerous and well documented. Reviews are given in standard books $[89,90]$. For $\mathrm{Pb}$ clusters, the size-induced drop of $T_{m}(R)$ is clearly recovered by simulations (Fig. 24). For Au clusters, of special experimental interest, MD simulations were carried out in order to understand how the premelted state was reached for increasing temperature. It was found first of all that the experimental size-driven lowering of $T_{m}(R)$ for Au clusters is confirmed. It can be seen in Figs. 25 and 26, that liquid-like diffusivity sets in at the surface below the cluster melting temperature (unless the cluster is so small as to melt at $T_{m}(\infty) / 2$ or below). Melting at $T_{m}(R)$ occurs by a sudden propagation of this liquid "skin" into the bulk-like interior. This behavior is quite similar to that described by a phenomenological model of Celestini et al [91].

One would expect that clusters entirely bounded by NM crystal faces should not premelt. While some overheating has been confirmed in $\mathrm{Pb}$ clusters bounded entirely by (111) facets [57], this is not generally true. For example, clusters of systems with $H<0$ such as In [94] have been shown to premelt. The problem is that Eq. (10) is macroscopic, and as such it ignores microscopic details, such as edges and corners that clusters nonetheless possess.

The simulated high temperature behavior of a $\mathrm{NaCl}$ cubic nanocluster ( a "nanograin of salt") is quite revealing [96]. The (100) nanocube faces are NM, and if they were unbounded, there would indeed be no premelting. However, the cube corners are the weak spot, and begin to melt even below $T_{m}$. NaCl corner roughening was described long ago [95] however in a rigid step model, that did not permit melting. It remains in principle an open question how the picture should evolve with increasing grain size. Clearly the corners will 
represent in all cases point-like germs of the liquid which are present below $T_{m}$. While these germs will certainly enlarge into liquid pools, it remains to be clarified how the pools will spread out, and if and in what form their presence will permit, the faces of a large $\mathrm{NaCl}$ cube to exhibit $\mathrm{NM}$ and to remain solid up to and above $T_{m}$. More generally, this approach should probably shed light also on the premelting of nanoparticles made of materials with $H<0$ mentioned above.

\section{Summary}

We reviewed the high temperature behavior of solid surfaces, in particular their tendency to wet spontaneously close to the bulk melting point. The case for which this does not happen ( and the melt somewhat unexpectedly fails to wet its own solid - surface nonmelting) has been treated in considerable detail. The large variety of behavior shown by different classes of solids has been addressed. We have also discussed other surface melting phenomena including rotational melting, glass surface defreezing, premelting of nanosystems. Finally, the existence of order at liquid surfaces has been highlighted as an important theme that is attracting increasing interest. It is hoped that this panoramic overview, despite its deliberate conciseness, will be of interest not only to the physics community, where it originates, but also to the materials science and chemical physics community, where it might be of some use.

\section{Acknowledgments}

This project was sponsored by Italian Ministry of University and Research, through COFIN2003, COFIN2004, FIRB RBAU01LX5H, and FIRB RBAU017S8R; and by INFM, through PRA NANORUB and "Iniziativa Trasversale calcolo parallelo". A large fraction of the calculations reviewed here were performed at CINECA, Casalecchio (Bologna). 


\section{References}

[1] J. W. M. Frenken, P. M. J. Marèe, and J. F. van der Veen, Phys. Rev. B 34 (1986), 7506.

[2] P. Carnevali, F. Ercolessi, and E. Tosatti, Phys. Rev. B 36 (1987), 6701.

[3] B. Pluis, A. W. Denier van der Gon, J. W. M. Frenken, and J. F. van der Veen, Phys. Rev. Lett. 59 (1987), 2678.

[4] S. Dietrich, in Transitions and critical phenomena, eds C. Domb and J. Lebowitz (Academic, London, 1987), Vol.12.

[5] F. D. Di Tolla, F. Ercolessi, and E. Tosatti, Phys. Rev. Lett. 74 (1995), 3201.

[6] O. Tomagnini, F. Ercolessi, S. Iarlori, F.D. di Tolla and E.Tosatti, Phys. Rev. Lett. 76 (1996), 1118.

[7] L. D. Landau and E. M. Lifshitz, Statistical Physics, (Pergamon, Oxford, 1980), Ch.XV

[8] B. Pluis, T. N. Taylor, D. Frenkel, and J. F. van der Veen, Phys. Rev. B 40 (1989), 1353.

[9] T. Zykova-Timan, U. Tartaglino, D. Ceresoli and E. Tosatti, accepted to PRL.

[10] see, e.g. J. P. Hansen and I. R. McDonald, Theory of Simple Liquids, (Academic Press, London, 1986).

[11] P. M. Platzman and H. Fukuyama, Phys. Rev. B 10 (1974), 3150.

[12] L. Pietronero and E. Tosatti, Solid State Commun. 32 (1979), 255.

[13] G. Tammann, Z. Phys. Chem.68 (1910), 205.

[14] I. N. Stranski, Z. Phys.119 (1942), 22.

[15] D. Nenow, Progress in Crystal Growth and Characterization (Pergamon, Oxford, 1984), Vol. 9, p.185.

[16] C. S. Jayanthi, E. Tosatti, and A. Fasolino, Phys. Rev. B 31 (1985), 470.

[17] A. M. Molenbroek and J. W. M. Frenken, Phys. Rev. B 50 (1994), 11132.

[18] R. Lipowsky and W. Speth, Phys. Rev. B 28 (1983), 3983.

[19] A. Trayanov and E. Tosatti, Phys. Rev. Lett. 59 (1987), 2207.

[20] J. N. Israelachvili, Intermolecular and surface forces (Academic Press, San Diego, 1985).

[21] J. G. Dash, Contemp. Phys. 30 (1989), 89.

[22] J. G. Dash, Rep. Prog. Phys. 58 (1995), 115. 
[23] A. A. Chernov and L. V. Mikheev, Phys. Rev. Lett. 60 (1988), 2488.

[24] X. J. Chen, F. Ercolessi, A. C. Levi, and E. Tosatti, Surf. Sci. 264 (1992), 207.

[25] M. S. Daw and M. I. Baskes, Phys. Rev. B 29 (1984), 6443.

[26] F. Ercolessi, M. Parrinello, and E. Tosatti, Phil. Mag. A 58 (1988), 213.

[27] M. W. Finnis and J. E. Sinclair, Phil. Mag. A 50 (1984), 45.

[28] D. W. Brenner, Phys. Rev. B 42 (1990), 9458.

[29] J. Tersoff, Phys. Rev. B 37 (1988), 6991.

[30] N. Takeuchi, A. Selloni, and E. Tosatti, Phys. Rev. Lett. 72 (1994), 2227.

[31] F. Di Tolla, E. Tosatti, and F. Ercolessi, in "Monte Carlo and Molecular Dynamics of Condensed Matter Systems", eds K. Binder and G. Ciccotti, Società (Italiana di Fisica, Bologna, 1996), p.345.

[32] J. G. Broughton and L. V. Woodcock, Solid State Phys. 11 (1978), 2743.

[33] J. G. Broughton and G. H. Gilmer, J. Chem. Phys. 79 (1983), 5105.

[34] see F.Y.Hansen, H.Taub, in "Phase Transitions in Surface Films 2", eds. G. Torzo, H.J Lauter and S.C. Fain, Jr. (NATO ASI Series,Series B: Physics, Plenum Publishing Corporation, New York, v.267).

[35] For a review, see e.g. M. Bernasconi and E. Tosatti, Surf. Sci. Rep., 17 (1993), 363.

[36] A. C. Levi and E. Tosatti, Surf. Sci 178 (1986), 425.

[37] M. den Nijs in Phase Transitions in Surface Films 2, eds H. Taub et al (NATO - ASI Series B, New York, 1991), Vol. 267, p. 247.

[38] K. Rommelse and M. den Nijs, Phys. Rev. Lett. 59 (1987), 2578.

[39] G. Santoro, M. Vendruscolo, S. Prestipino, and E. Tosatti, Phys. Rev. B 53 (1996), 13169.

[40] S. Prestipino, G. Santoro, and E. Tosatti, Phys. Rev. Lett. 75 (1995), 4468.

[41] H. S. Youn and G. B. Hess, Phys. Rev. Lett. 64 (1990), 918.

[42] H. S. Youn, X. F. Meng, and G. B. Hess, Phys. Rev. B 48 (1993), 14556.

[43] M. den Nijs, Phys. Rev. Lett. 66 (1991), 907.

[44] J. M. Phillips, Q. M. Zhang, and J. Z. Larese, Phys. Rev. Lett. 71 (1993), 2971.

[45] E. A. Jagla, S. Prestipino, and E. Tosatti, Phys. Rev. Lett. 83 (1999), 2753.

[46] F. Celestini, D. Passerone, F. Ercolessi, and E. Tosatti, Surf. Sci. 402 (1998), 886. 
[47] F. Celestini, D. Passerone, F. Ercolessi, and E. Tosatti, Phys. Rev. Lett. 84 (2000), 2203.

[48] C. S. Jayanthi, F. Celestini, F. Ercolessi, and E. Tosatti, Surf. Sci. 460 (2000), L503.

[49] G. Grange and B. Mutaftschiev, Surf. Sci. 47 (1975), 723; L. Komunjer, D. Clausse and B. Mutaftschiev, J. Cryst. Growth 182, 205 (1997).

[50] T. Zykova-Timan, U. Tartaglino, D. Ceresoli, W. Zaoui-Sekkal and E. Tosatti, Surf. Sci. 566/568 (2004), 794.

[51] R. Trittibach, Ch. Grütter, and J. H. Bilgram, Phys. Rev. B 50 (1994), 2529.

[52] J. F. van der Veen in Phase Transitions in Surface Films 2, eds H. Taub et al (NATO - ASI Series B, New York, 1991), Vol. 267, p. 289.

[53] S. Modesti, V. R. Dhanak, M. Sancrotti, A. Santoni, B. N. J. Persson, and E. Tosatti, Phys. Rev. Lett. 73 (1994), 1951.

[54] R. Car and M. Parrinello, Phys. Rev. Lett., 55 (1985), 2471.

[55] A. M. Molenbroek and J. W. Frenken, Phys. Rev. B 50 (1994), 11132.

[56] J. W. Herman and H. E. Elsayed-Ali, Phys. Rev. Lett. 74 (1995), 3201.

[57] J. J. Métois and J. C. Heyraud, J. Phys.(France) 50 (1989), 3175.

[58] P. Noziéres, J. Phys. (Paris) 50 (1989), 2541.

[59] G. Bilalbegović, F. Ercolessi, and E. Tosatti, Europhys. Lett. 17 (1992), 333.

[60] H. M. van Pinxteren, B. Pluis, and J. W. M. Frenken, Phys. Rev. B 49 (1994), 13798.

[61] R. H. Torii and S. Balibar, Physica B 194 (1994), 971.

[62] U. Tartaglino and E. Tosatti, Surf. Sci. 532-535 (2003), 623.

[63] U. Tartaglino and E. Tosatti, to be published.

[64] R. J. Asaro and W. A. Tiller, Metall. Trans. 3 (1972), 1789.

[65] M. Grinfeld, J. of Phys.: Condens. Matter 4 (1992), L647.

[66] H. Ibach, Surf. Sci. Rep. 29 (1997), 193.

[67] P. W. Anderson, Basic notions of condensed matter physics, (Menlo Park, Calif. : Benjamin/Cummings, 1984), Ch.4.

[68] R. J. Speedy, Mol. Phys. 95 (1998), 169.

[69] R. J. Speedy, J. Phys. Chem. B 1023 (1999), 4061.

[70] E. A. Jagla and E. Tosatti, Europhys. Lett. 51 (2000), 648.

[71] Y. M. Stokes Proc. R. Soc. London Ser. A 455 (1999), 2751. 
[72] E. A. Jagla and E. Tosatti, Surface Sci., 464 (2000), L686.

[73] P. A. Heiney,J. E. Fischer, A. R. McGhie, W. J. Romanow, A. M. Denenstein, J. P. McCauley Jr. and A. B. Smith, Phys. Rev. Lett. 66 (1991), 2911.

[74] D. Passerone and E. Tosatti, Surf. Rev. and Lett. 4 (1997), 859.

[75] A. Goldoni, C. Cepek, and S. Modesti Phys. Rev. B 54 (1996), 2890.

[76] C. Laforge, D. Passerone, A. B. Harris, P. Lambin, and E. Tosatti, Phys. Rev. Lett. 87 (2001), 085503.

[77] see, e.g., H. Tostmann, E. DiMasi, P. S. Pershan, B. M. Ocko, O. G. Shpyrko, and M. Deutsch, Phys. Rev. B 61 (2000), 7284 and references therein.

[78] O. Shpyrko, M. Fukuto, P. Pershan, B. Ocko, I. Kuzmenko, T. Gog and M. Deutsch, Phys. Rev. B 69 (2004), 245423 and references therein.

[79] H. L. Lemberg, S. A. Rice, and D. Guidotti, Phys. Rev. B 10 (1974), 4079.

[80] N. H. March and M. P. Tosi, Atomic dynamics in liquids (Macmillan, London, 1976).

[81] X. Z. Wu, E. B. Sirota, S. K. Sinha, B. M. Ocko, and M. Deutsch, Phys. Rev. Lett. 70 (1993), 958.

[82] X. Z. Wu, B. M. Ocko, E. B. Sirota, S. K. Sinha, M. Deutsch, B. M. Cao, and M. W. Kim, Science 261 (1993), 1018.

[83] D. R. Nelson and B. I. Halperin, Phys. Rev. Lett. 41 (1978), 121.

[84] C. A. Murray and R. A. Wenk, Phys. Rev. Lett. 62 (1989), 1643.

[85] D. Passerone, F. Ercolessi, F. Celestini and E. Tosatti, Surf. Rev. Lett. 6 (1999), 663.

[86] F. Celestini, F. Ercolessi and E. Tosatti, Phys. Rev. Lett. 78 (1997), 3153.

[87] Ph. Buffat and J. P. Borel, Phys. Rev. A 13 (1976), 2287.

[88] O. Gülseren, F. Ercolessi, and E. Tosatti, Phys. Rev. B 51 (1995), 7377.

[89] K. S. Liang, M. P. Anderson, R. F. Bruinsma, and G. Scoles, Interface dynamics and growth ( Pa.(MRS), Pittsburgh, 1992).

[90] V. Kumar, T. P. Martin, and E. Tosatti, in Clusters and fullerenes: proceedings of the Adriatico Research conference, Trieste, Italy, June 23-26, 1992 (World Scientific, Singapore, 1993).

[91] F. Celestini and A. Ten Bosch, Phys. Lett. A 207 (1995), 307.

[92] H. S. Lim, C. K. Ong, and F. Ercolessi, Zeitschrift für Physik D 26 (1993), S45.

[93] F. Ercolessi, W. Andreoni, and E. Tosatti, Phys. Rev. Lett. 66 (1991), 911.

[94] A. Pavlovska, D. Dobrev, and E. Bauer, Surf. Sci. 286 (1993), 176. 
[95] M. Wortis in Chemistry and physics of solid surfaces VIII , eds R. Vanselow, R. Howe (Springer-Verlag, Berlin, 1990).

[96] T. Zykova-Timan, U. Tartaglino, D. Ceresoli, and E. Tosatti, to be published.

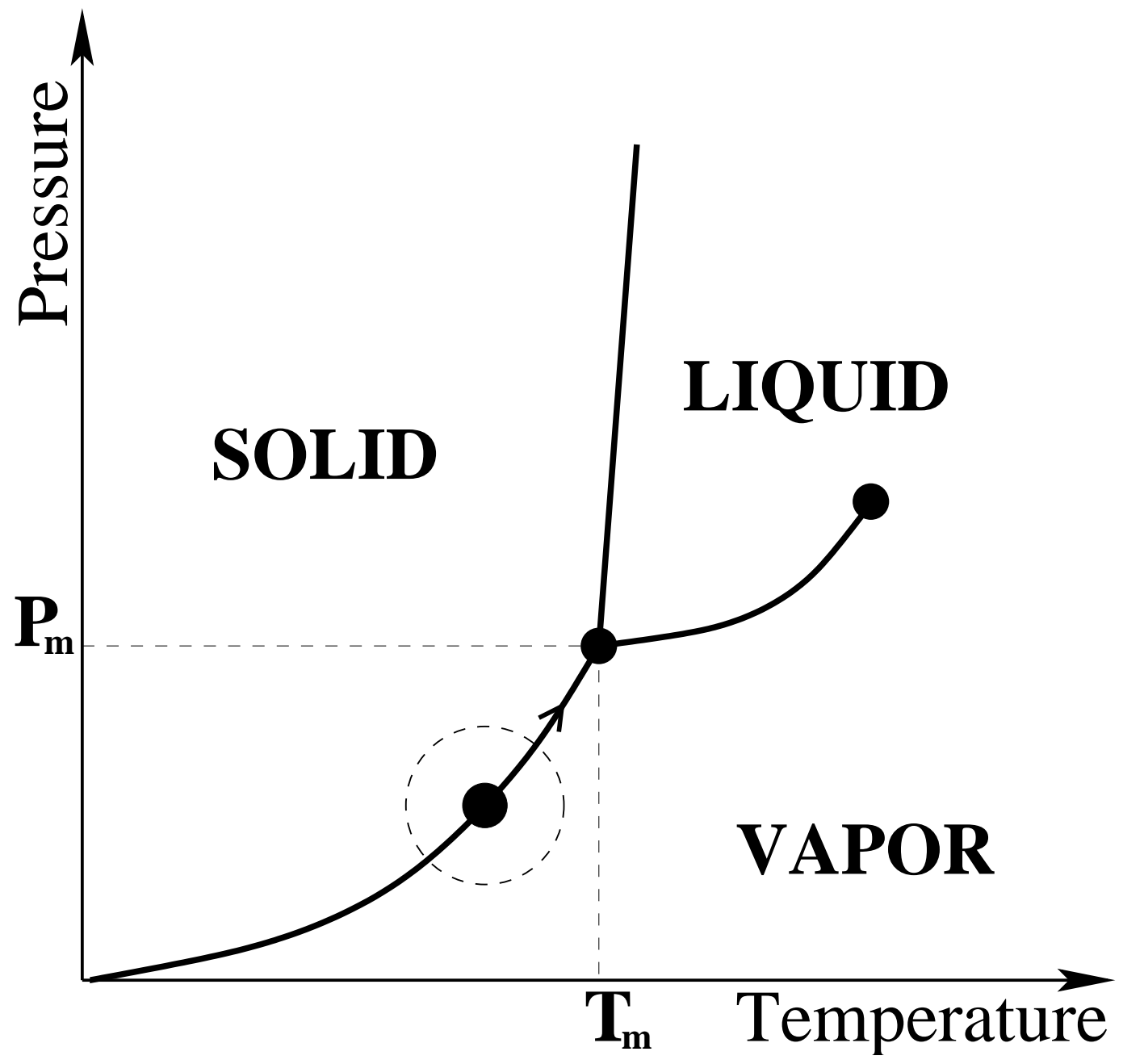

Fig. 1. Melting of a pure and homogeneous material by heating along the solid-vapor coexistence curve up to the triple point. 


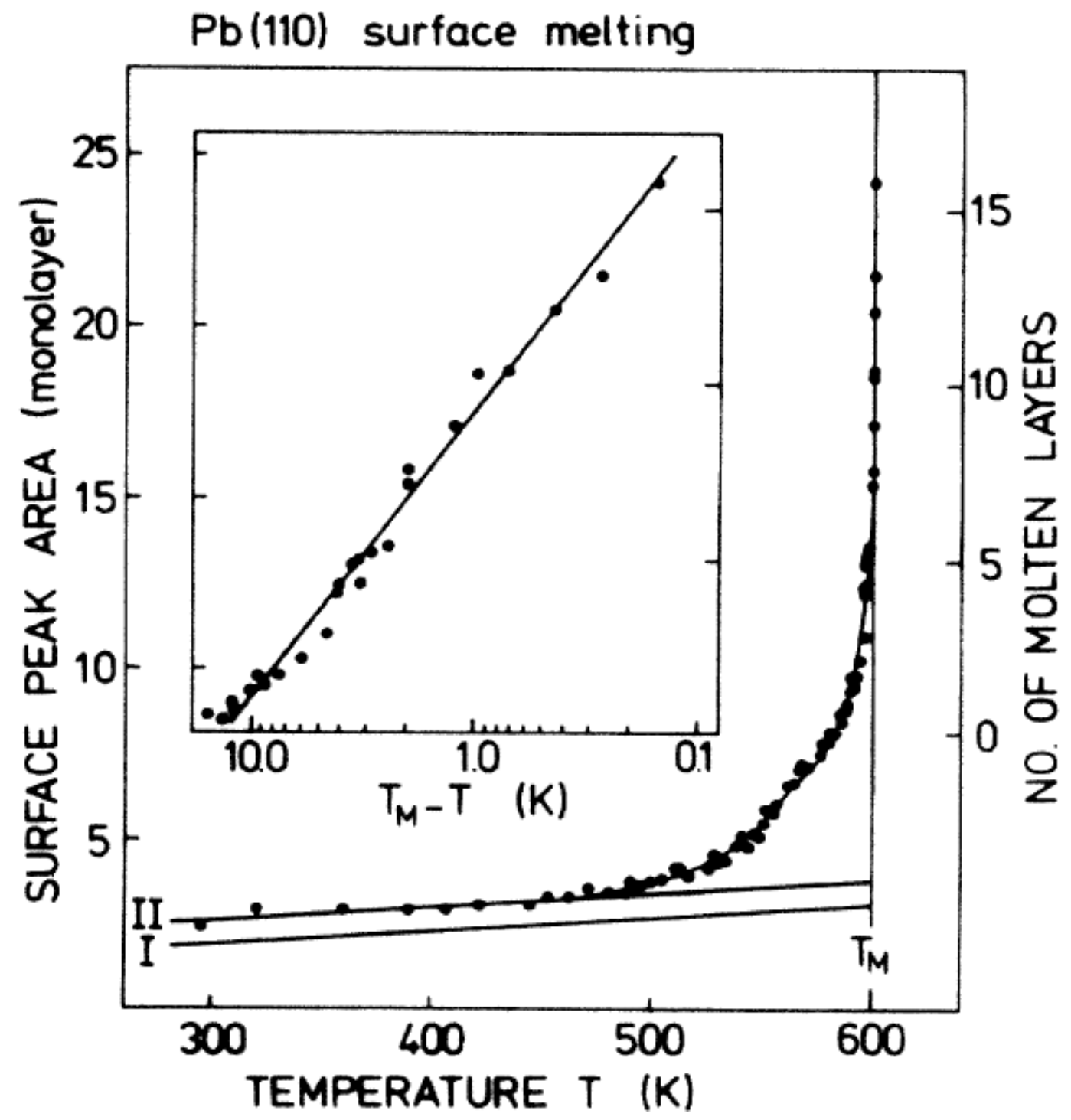

Fig. 2. Experimental evidence of surface melting of $\mathrm{Pb}(110)$ : Medium energy ion scattering shows an increasing number of disordered (liquid) layers approaching the melting temperature. From Ref. [1]. 


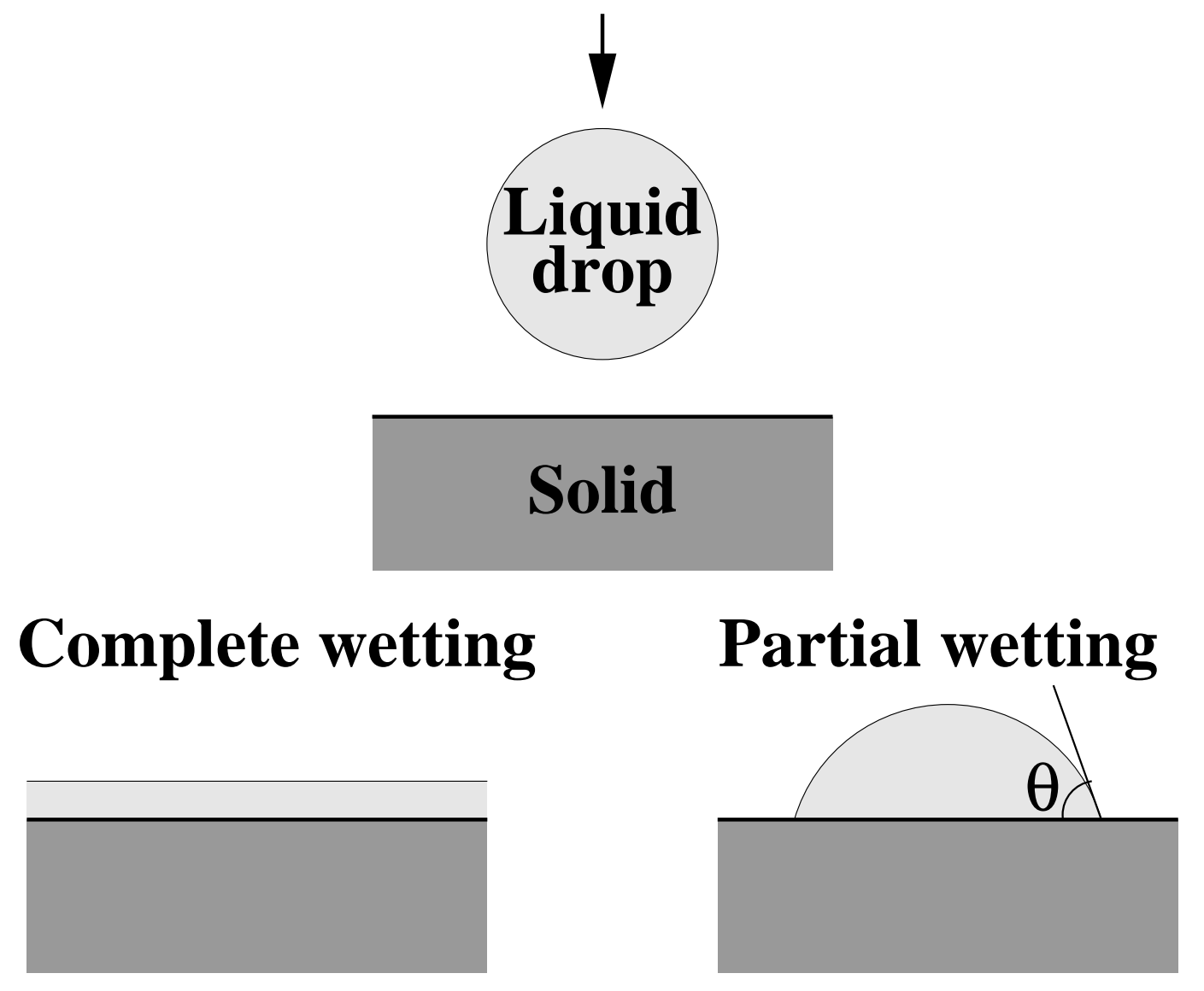

Fig. 3. Partial wetting of a solid by its own melt is an indirect evidence of surface nonmelting. 


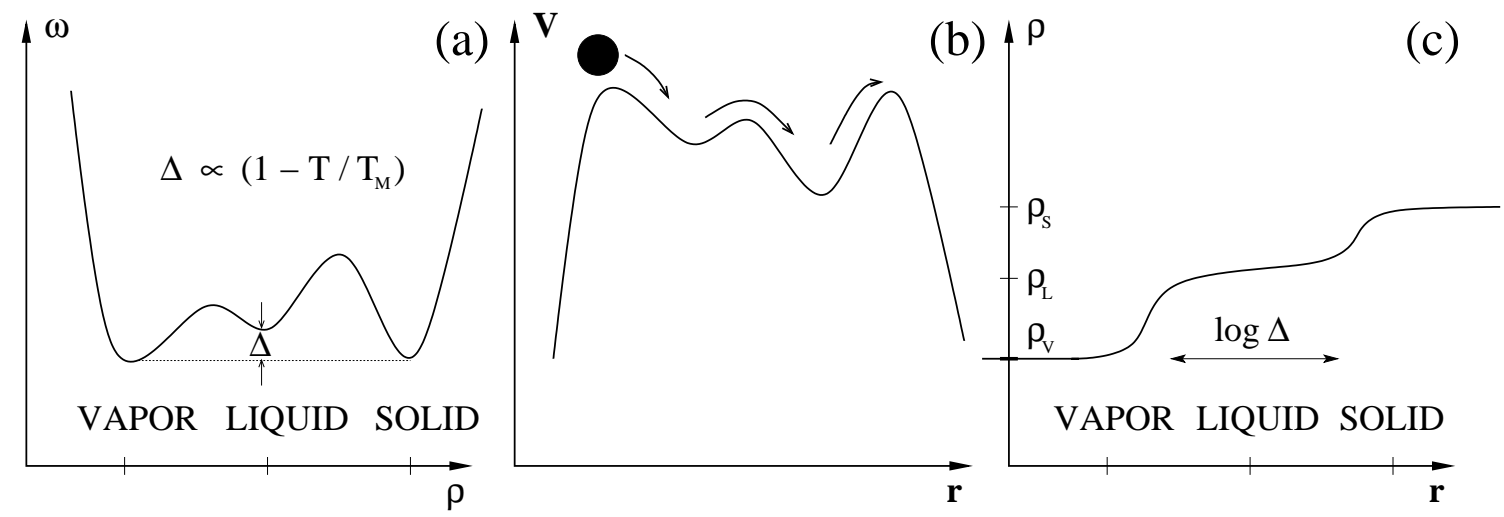

Fig. 4. (a) Minimizing grand potential (Eq.4), (b) Mechanical equivalent (see text), (c) Density profile, showing that the SV interface has split into a SL plus LV interfaces. 


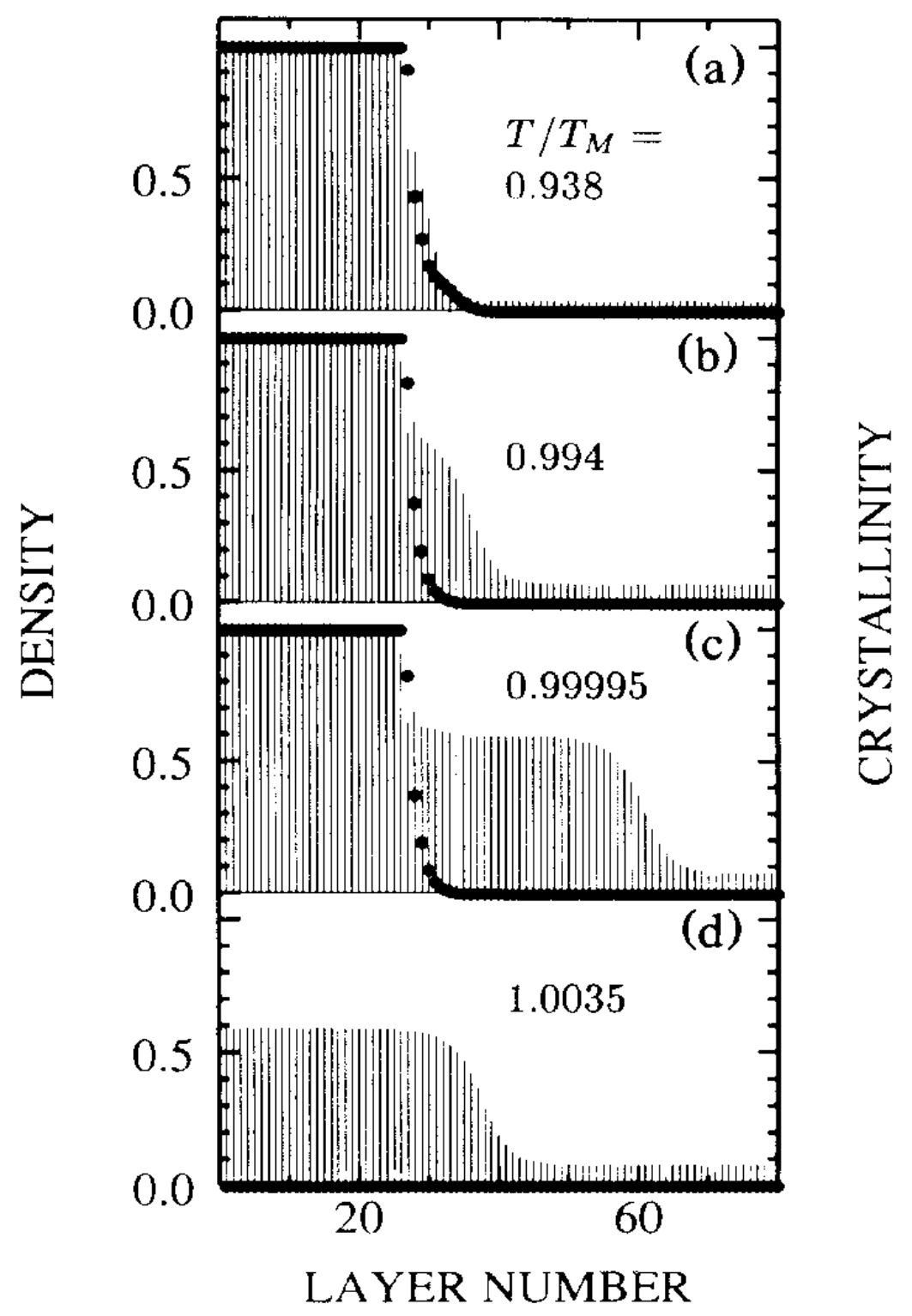

Fig. 5. Surface melting of LJ(110): density profile and cristallinity profile. From Ref. [19]. 


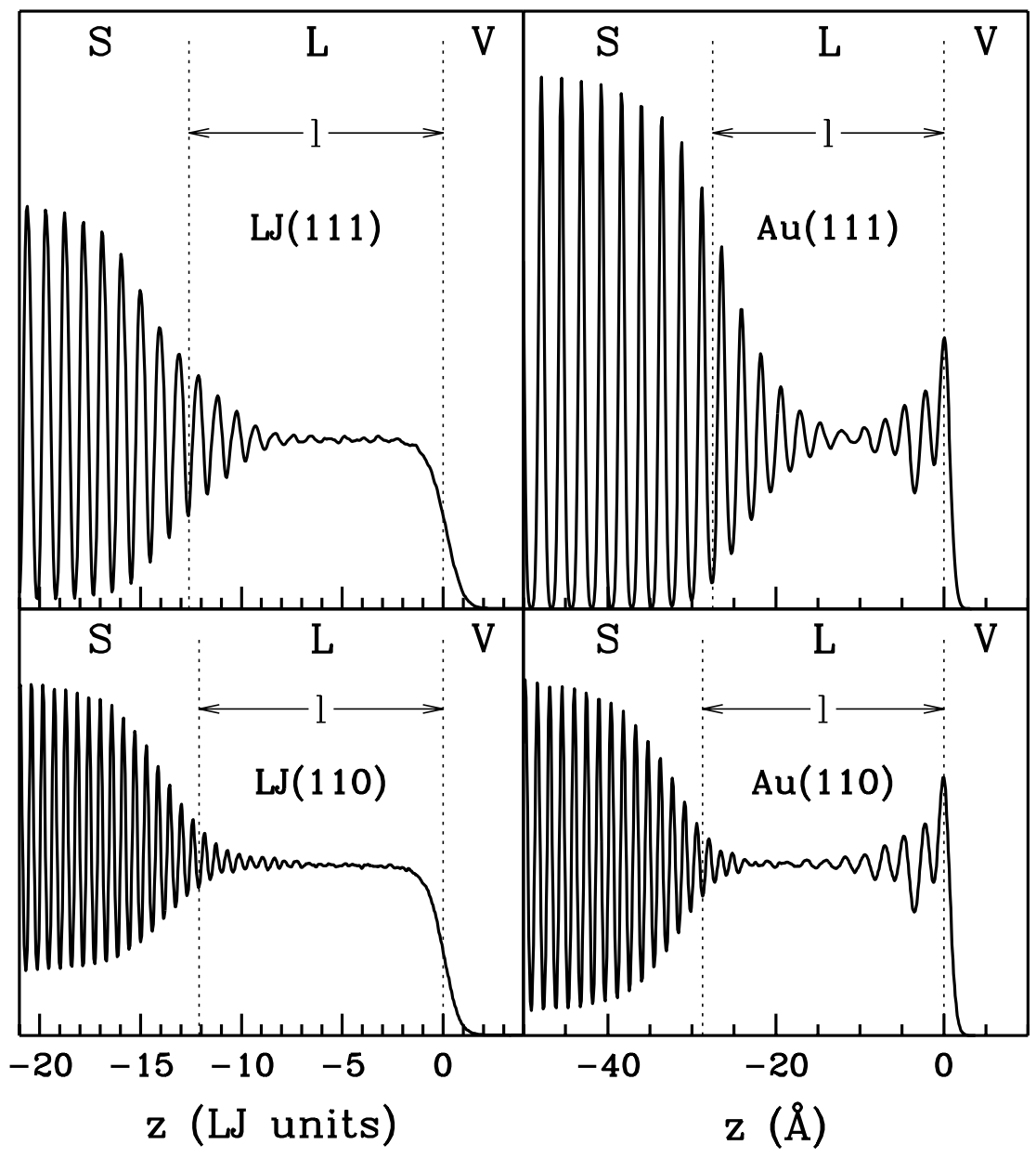

Fig. 6. Typical solid-liquid-vapor density profiles obtained by simulations of Lennard-Jones(110), (111) and $\mathrm{Au}(110)$ (three surfaces that undergo SM) just below $T_{m}$. For $\mathrm{Au}(111)$ (which is instead a NM surface) a nonequilibrium configuration is shown with two facing equal-period density oscillations which lead to attraction and eventually to the collapse of the two interfaces (see text). From Ref. [31]. 


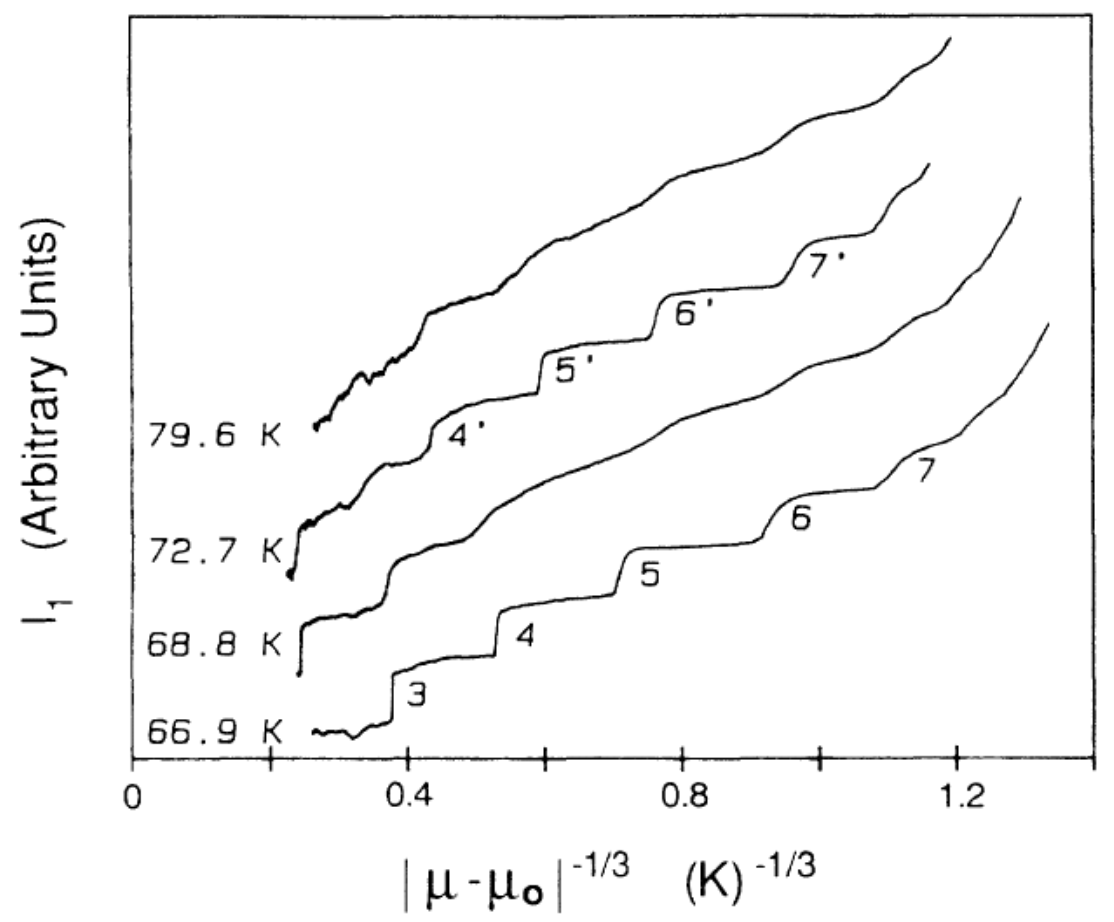

Fig. 7. Reentrant layering during the deposition of Argon on a graphite substrate. On the increasing $T$, the ellipsometric coverage isoterms switch from a staircase profile (growth layer-by-layer) to a continous one at $68.8 \mathrm{~K}$, and then again to a staircase profile as temperature is further increased. The new structure consists of half layers, instead of full layers. Image and data from [41]. 


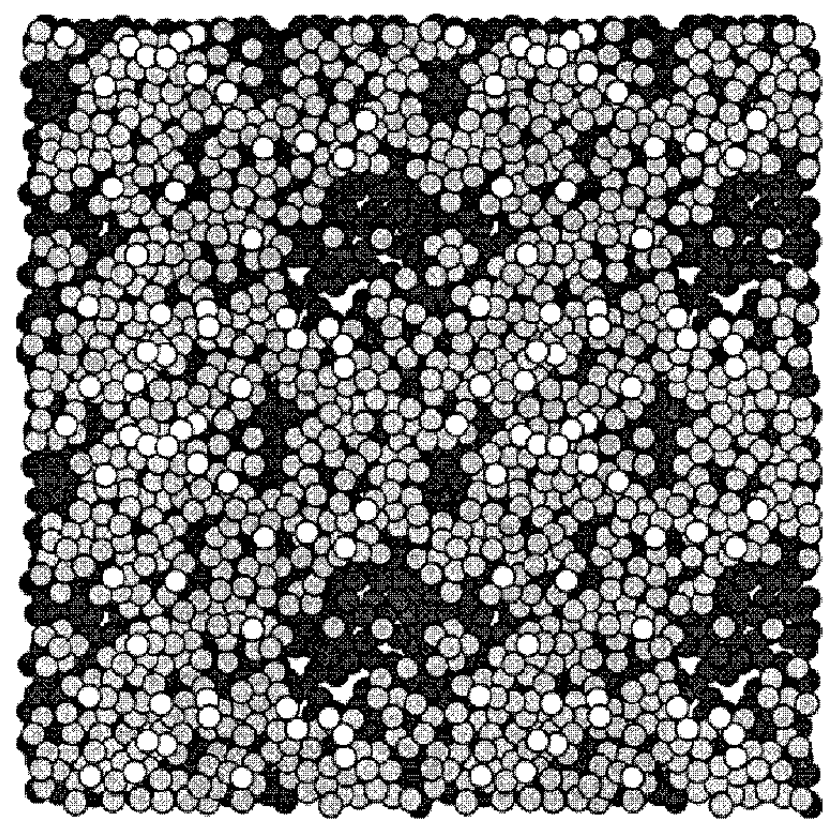

Fig. 8. Top view (snapshot) of the three outer layers of simulated grand canonical $\operatorname{Ar}(111)$ at $0.8 T_{m}$. This surface is nearly "disordered flat". Atoms are represented as black, grey or white if their $z$ coordinate indicates that they belong respectively to the subsurface layer, the surface layer or the adatom layer. For convenience, four adjacent simulation cells are shown. The nearly half occupancy of the surface layer (layer 1) is realized through large islands and large craters. Image from Ref. [46]. 


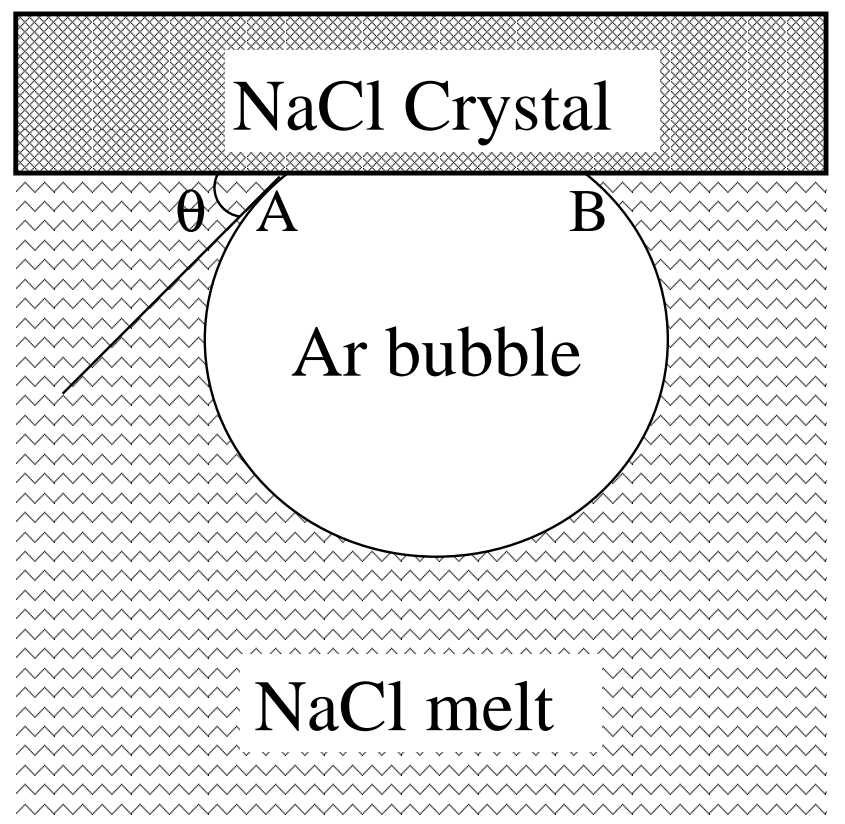

Fig. 9. Argon bubble studies of liquid $\mathrm{NaCl}$ in contact with the solid. The solid-liquid-vapor junctions A and B reveal a surprising lack of complete wetting, with a large partial wetting angle of about 48 degrees. 


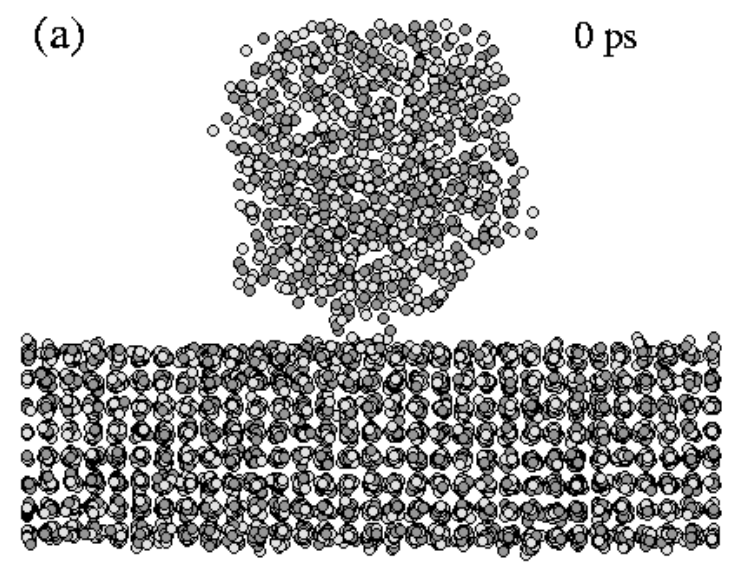

(c)

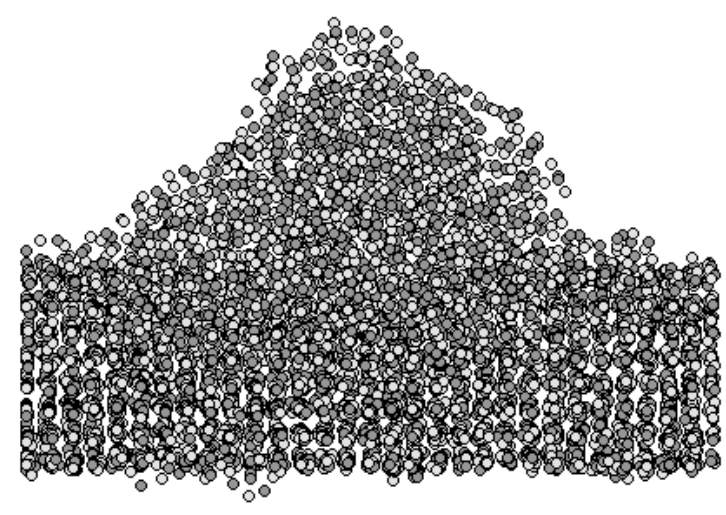

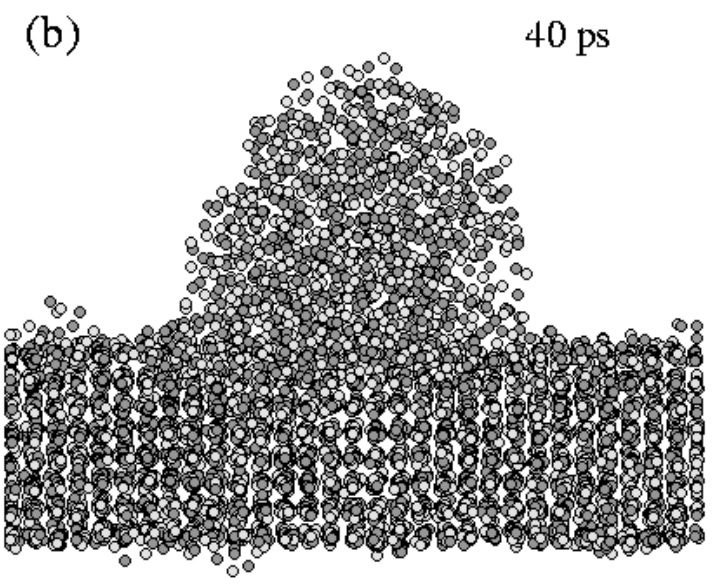

(d)

$200 \mathrm{ps}$

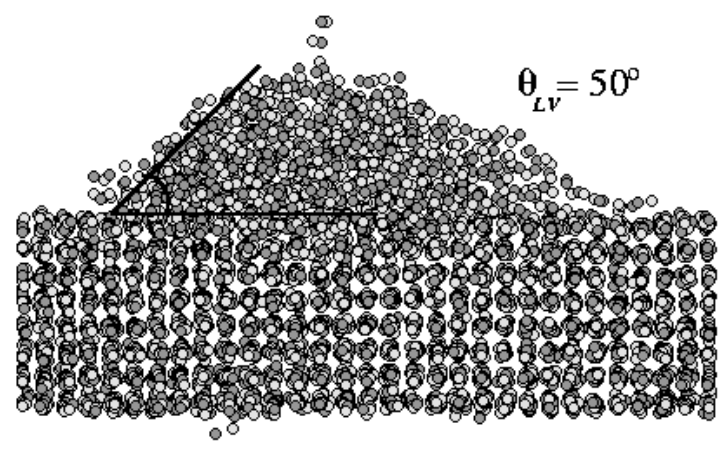

Fig. 10. The simulated time evolution of $\mathrm{NaCl}$ liquid nanodroplet brought into the contact with $\mathrm{NaCl}(100)$ at the melting point. After 100 ps the drop stabilizes in the metastable state forming a partial wetting contact angle $\theta=(50 \pm 5)^{\circ}$ From Ref. [50]. 


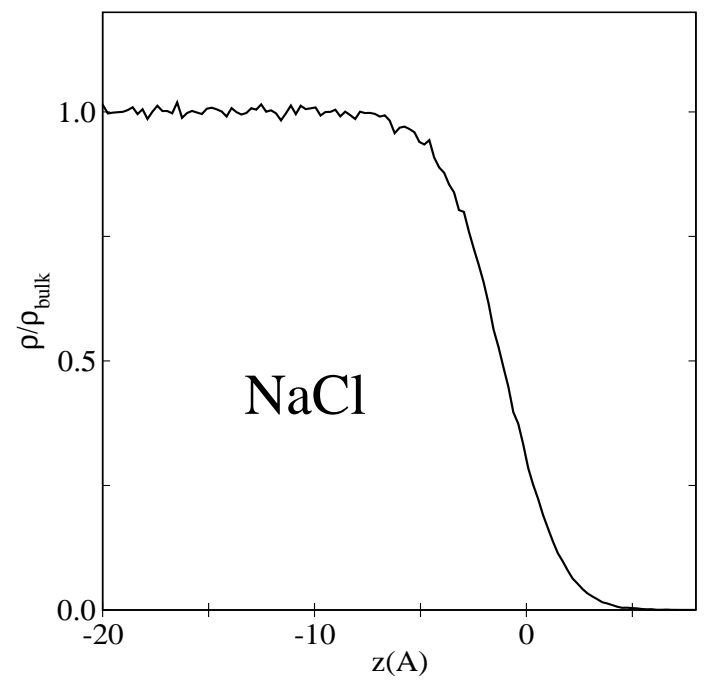

Fig. 11. Density profile of the liquid surface of $\mathrm{NaCl}$ obtained from a simulation at $T_{m}$. The density drops very smoothly from the bulk in the liquid phase to that of the vapor one. From Ref. [96].

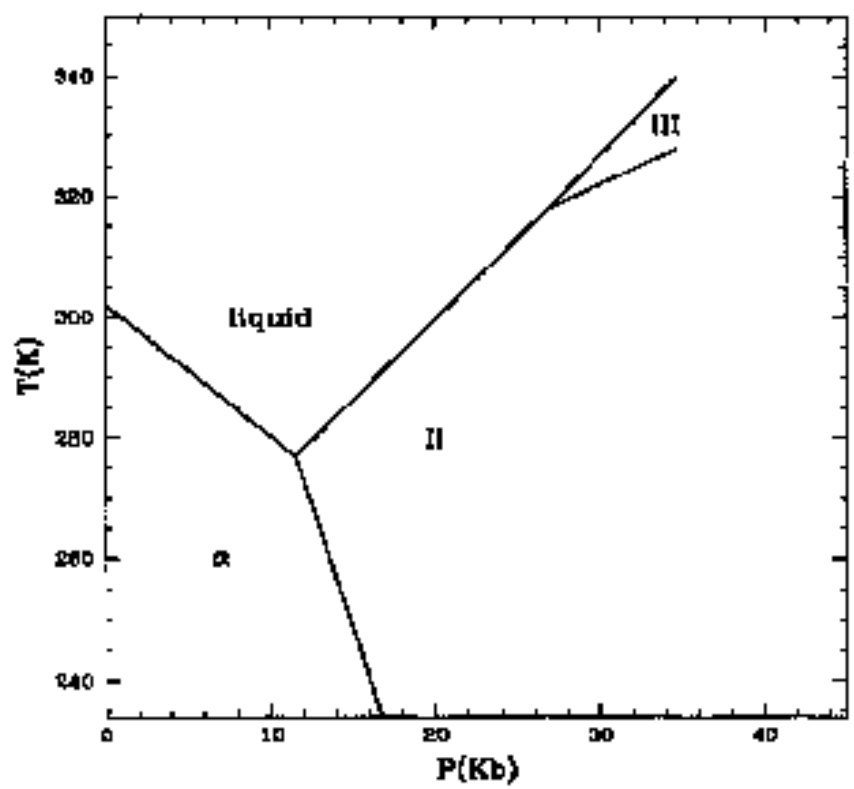

Fig. 12. Schematic Ga phase diagram, showing the semimetallic $\alpha$-phase surrounded by fully metallic liquid and solid-II phases. The picture for valence semiconductors such as $\mathrm{Si}, \mathrm{Ge}$ is similar. 


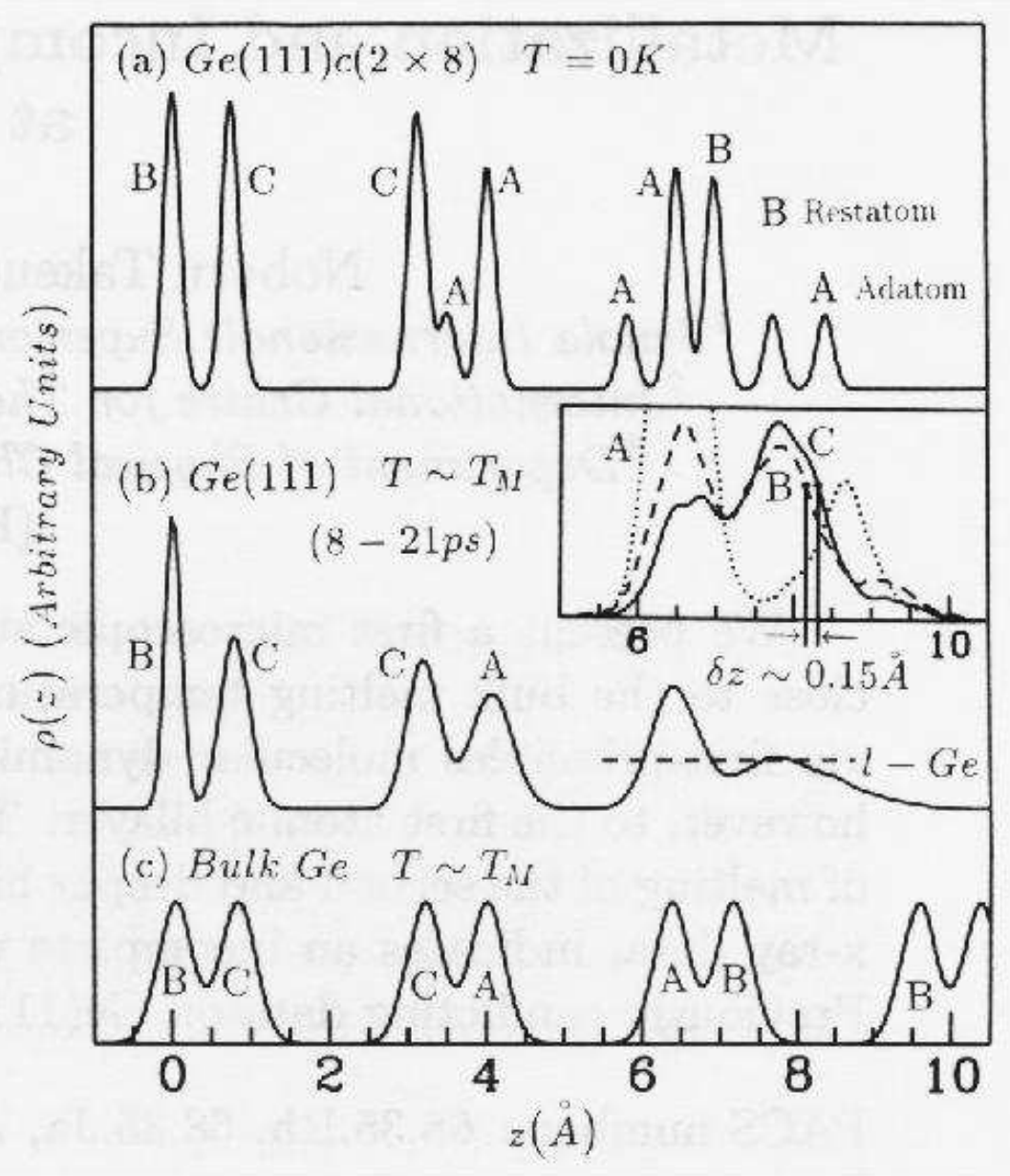

Fig. 13. $(x, y)$ averaged atom density profile for $(\mathrm{a})$ the $\mathrm{Ge}(111) c(2 \times 8)$ reconstructed surface at $T=0 \mathrm{~K}$, (b) the same Ge(111) surface at $T \sim T_{M}$, displaying "blocked melting" restricted to first bilayer and (c) crystalline bulk Ge at $T \sim T_{M}$. Note in (b) the disordering of the first bilayer with atoms distributed between different sublattices (inset) From Ref. [30]. 


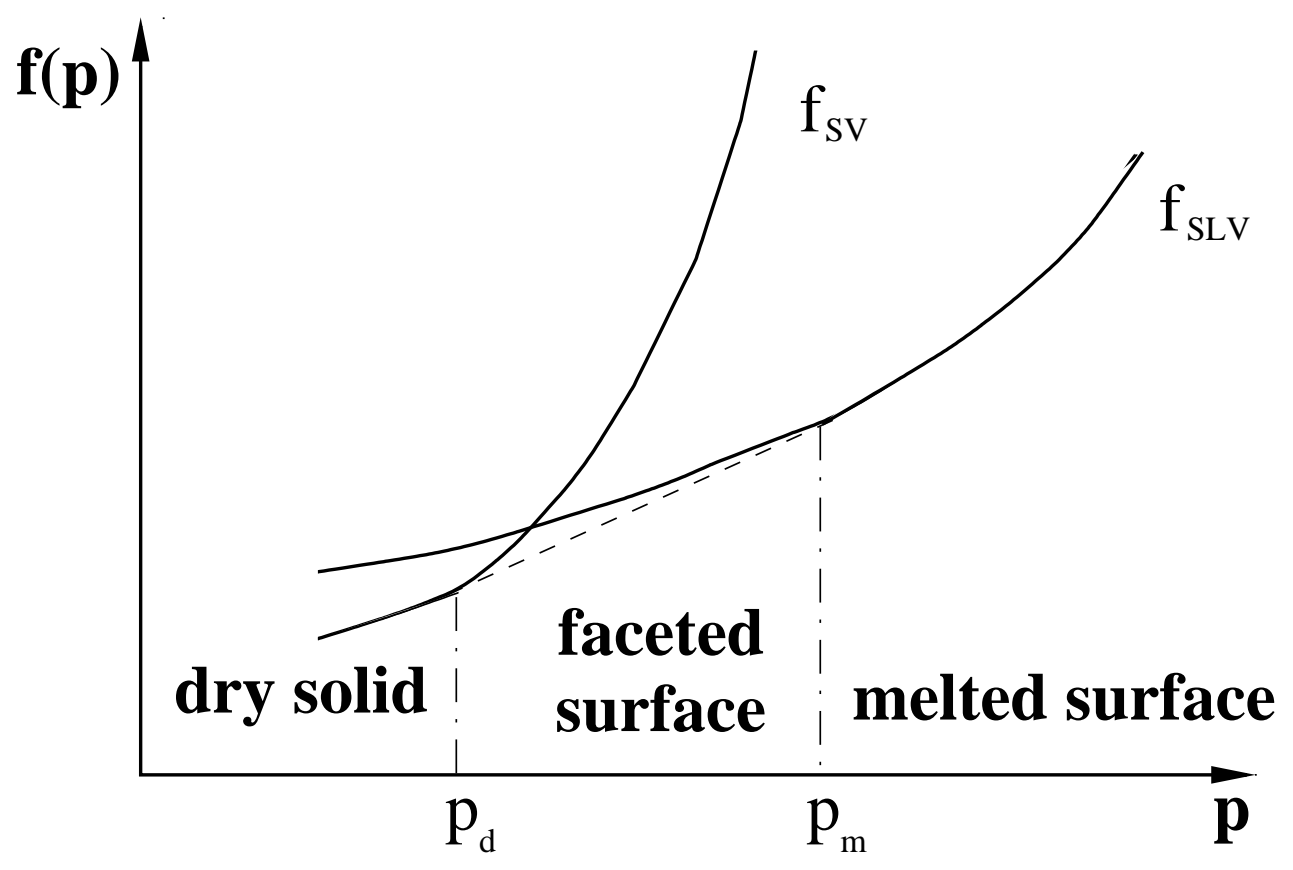

Fig. 14. Plot of a system with a solid (non-melting) branch and a liquid one. $p=\tan \theta$ and $f=\gamma / \cos \theta$, where $\theta$ is the tilting angle. The orientations up to $p_{d}$ remain $d r y$, in the range $\left(p_{d}, p_{m}\right)$ there is non-melting induced faceting, and above $p_{m}$ surface melting occurs. $p_{d}$ might coincide with 0. From Ref. [58]. 


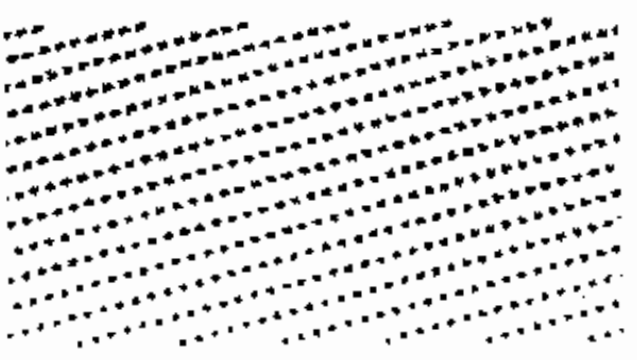

a)

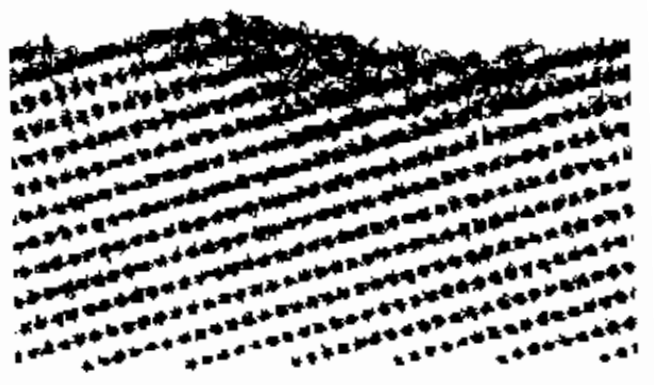

b)

Fig. 15. Non-melting induced faceting trajectories of the atoms in MD simulations, projected on a plane. (a) At low temperatures the $\mathrm{Pb}(432)$ surface is flat and stable. (b) At $0.97 T_{m}$ the surface is phase separated in $d r y$ and wet facets. The atoms in the liquid part are recognizable by their wandering trajectories. From Ref. [59]. 


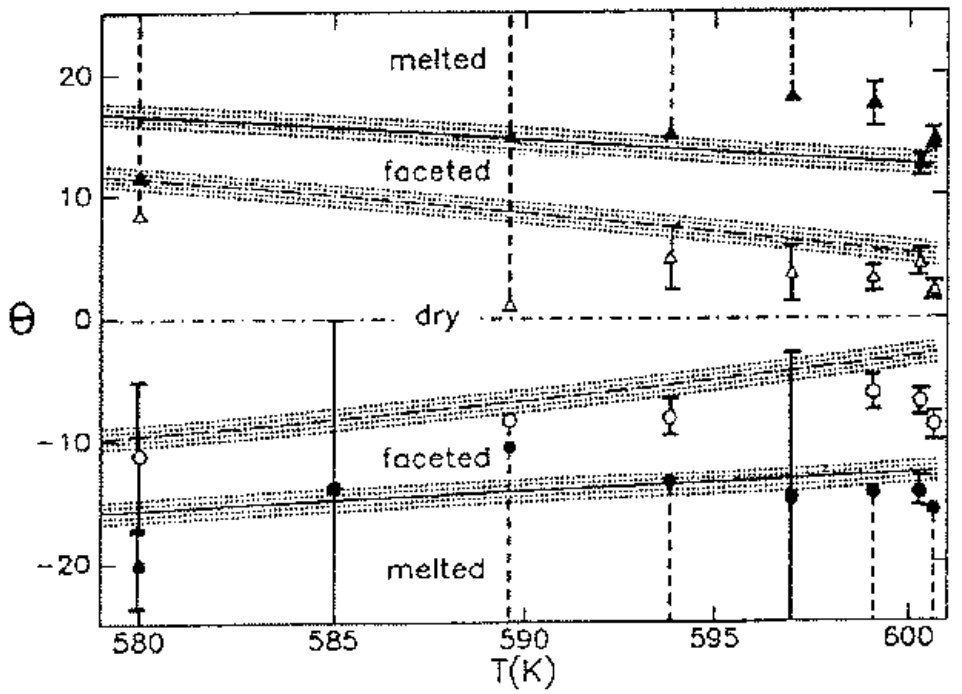

Fig. 16. Non-melting induced faceting of $\mathrm{Pb}(111)$ vicinals from Ref. [60]. Experimentally determined orientation angles as a function of $T$. Triangles refer to a tilt toward the (110) orientation, while circles refer to a tilt toward the (100) orientation. Open symbols refer to the angle below which the vicinal is dry, while filled symbols to the angle above which the surface is melted. We refer to the original work for an explanation of the fit (solid and dashed curved). 


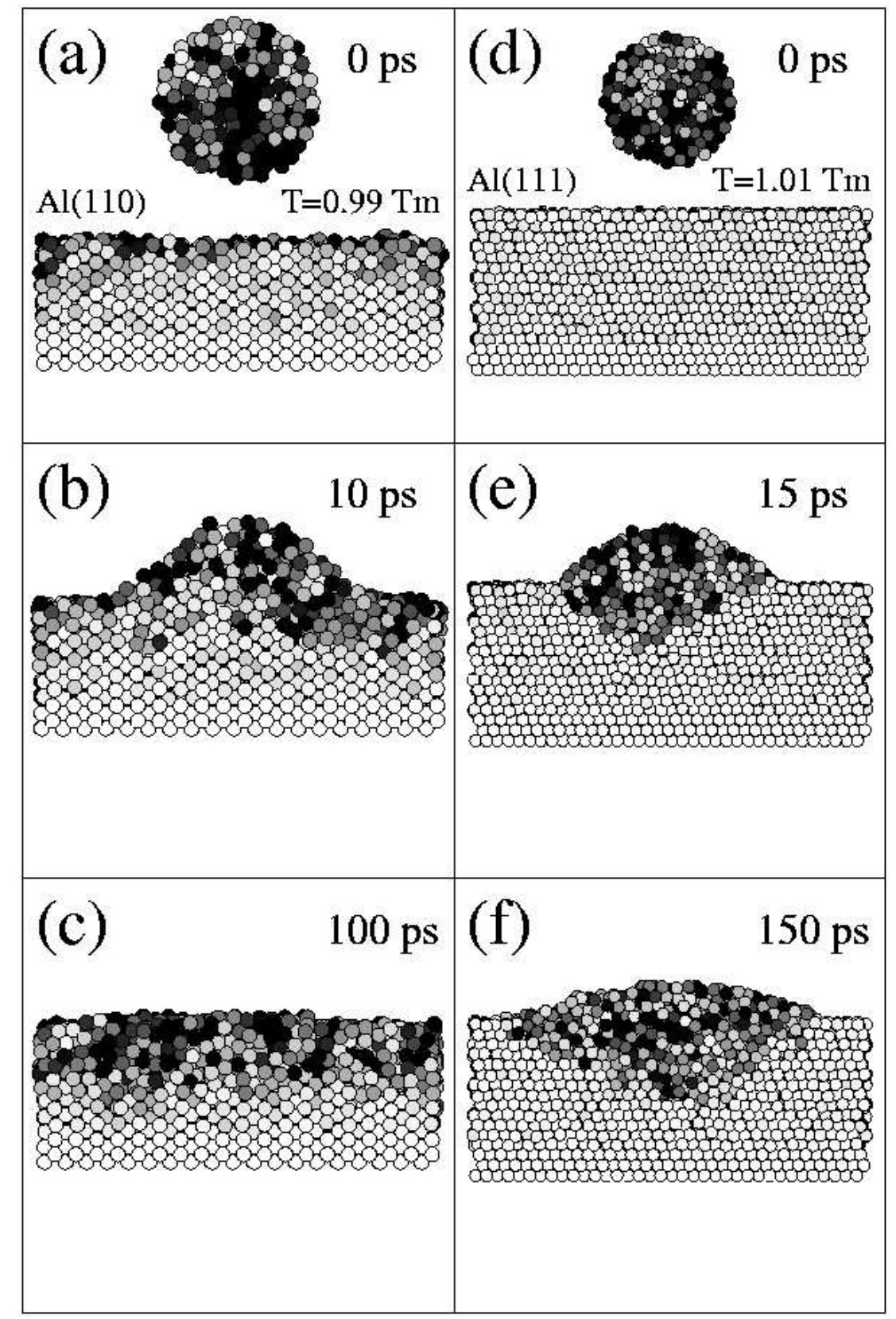

Fig. 17. Evolution of Al liquid drop on a surface of bulk Al. Left column: drop on a surface undergoing surface melting $\left(\mathrm{Al}(110)\right.$ at $\left.T=0.99 T_{m}\right)$. (a) before contact; (b) after contact, the drop spreads readily; (c) the drop has been fully absorbed. Right column: drop on a nonmelting overheated surface $\left(\mathrm{Al}(111)\right.$ at $\left.T=1.01 T_{m}\right)$. (d) before contact; (e) after contact: the drop settles but does not spread; (f) final drop shape. Darkness of atoms proportional to their square displacement in the run. 


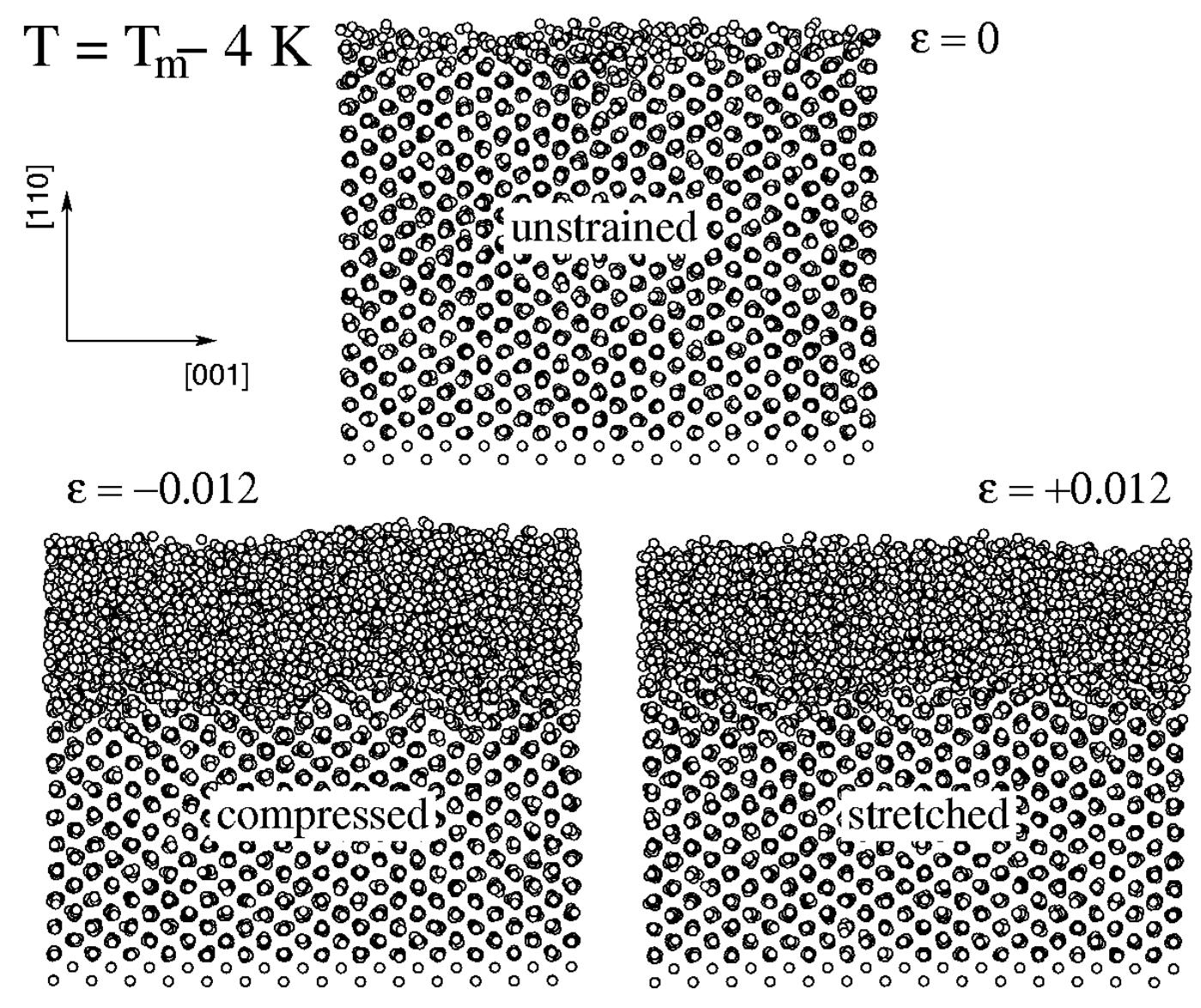

Fig. 18. Single snapshots (lateral view) of three simulated $\mathrm{Al}(110)$ surfaces at the same temperature but under different strain conditions. Samples' size: $14 \times 20 \times 16$. The atom stacking in the 20 -atom rows orthogonal to the picture clearly distinguishes the liquid and the solid phases. 


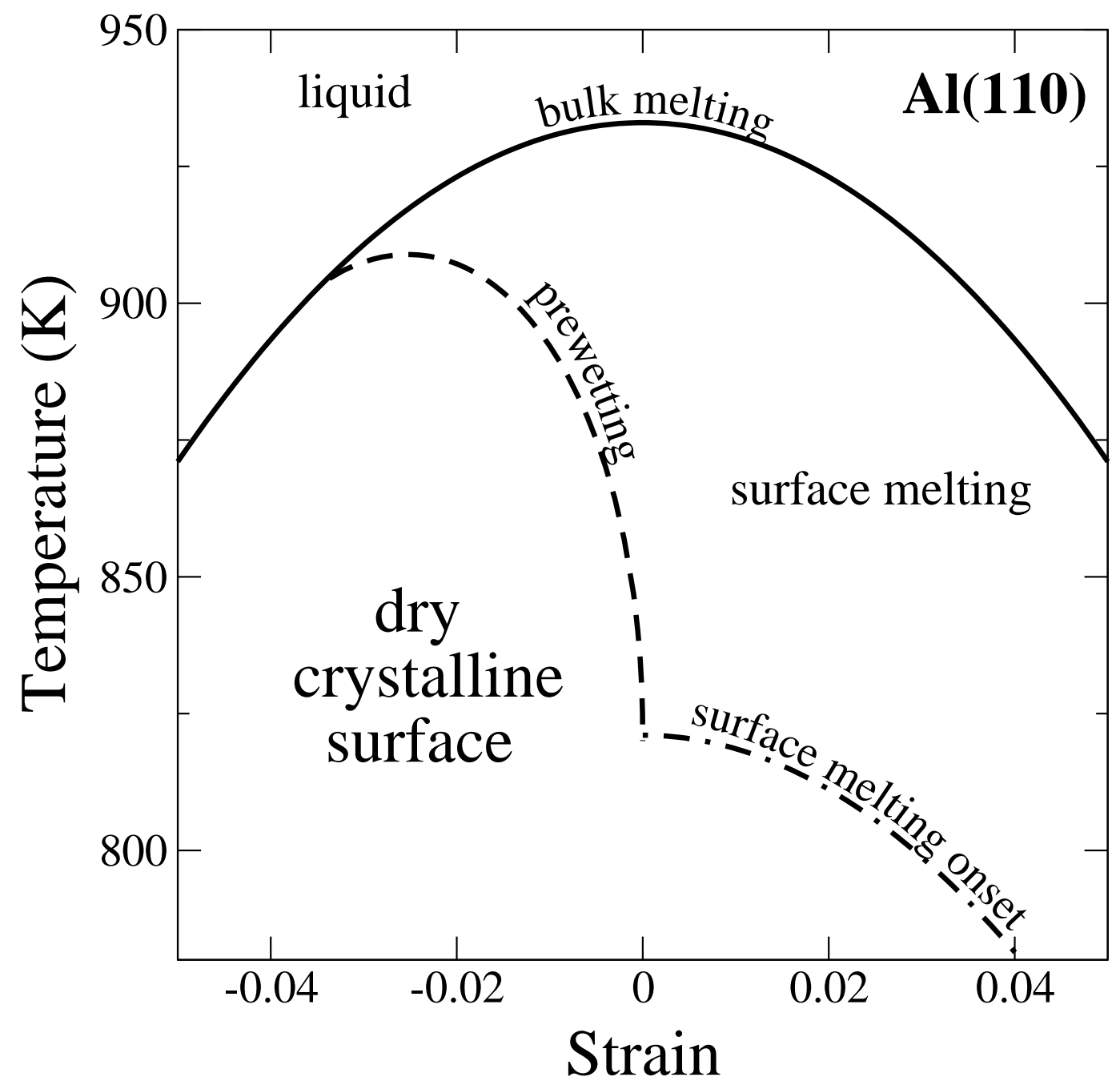

Fig. 19. Phase diagram of a metal surface in presence of in-plane strain. Note that the negative (compressive) strain can give rise to a prewetting transition. From Ref. [62]. 

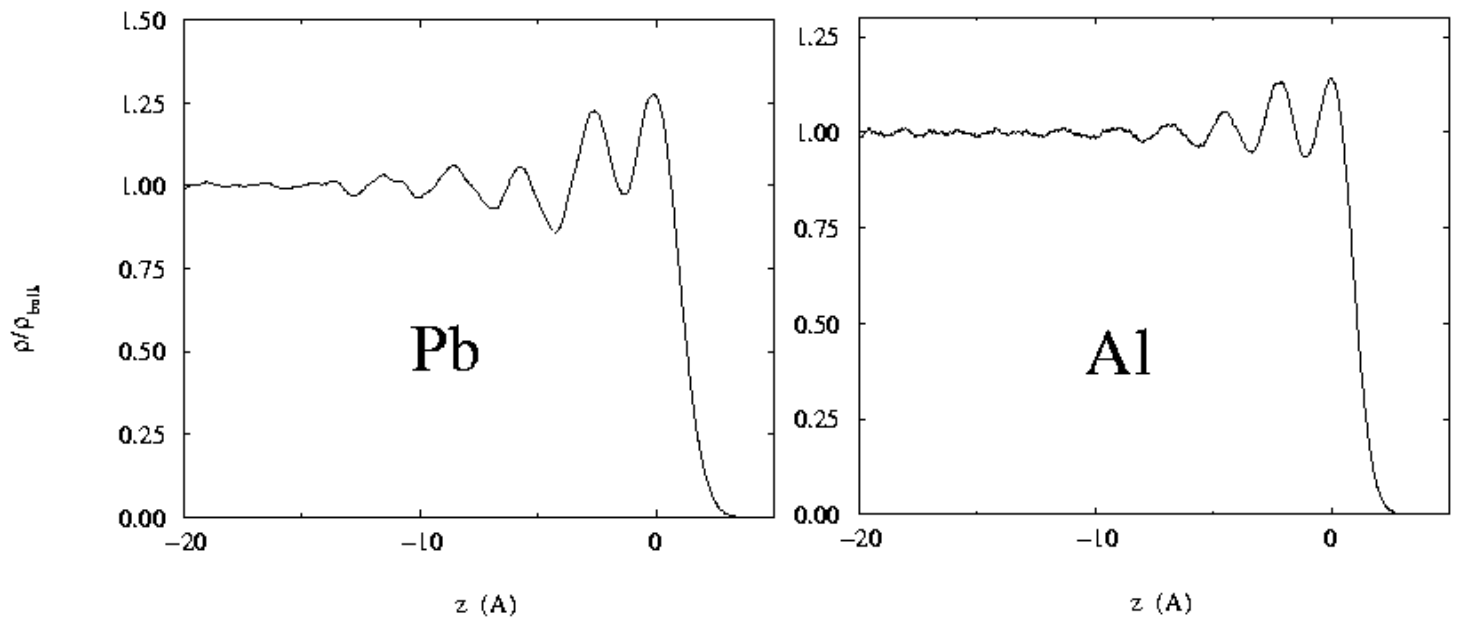

Fig. 20. Density profile of the liquid surface of $\mathrm{Pb}$ and $\mathrm{Al}$ obtained from a simulation at $T_{m}$. The profile shows layering oscillations at the liquid-vapor interface.
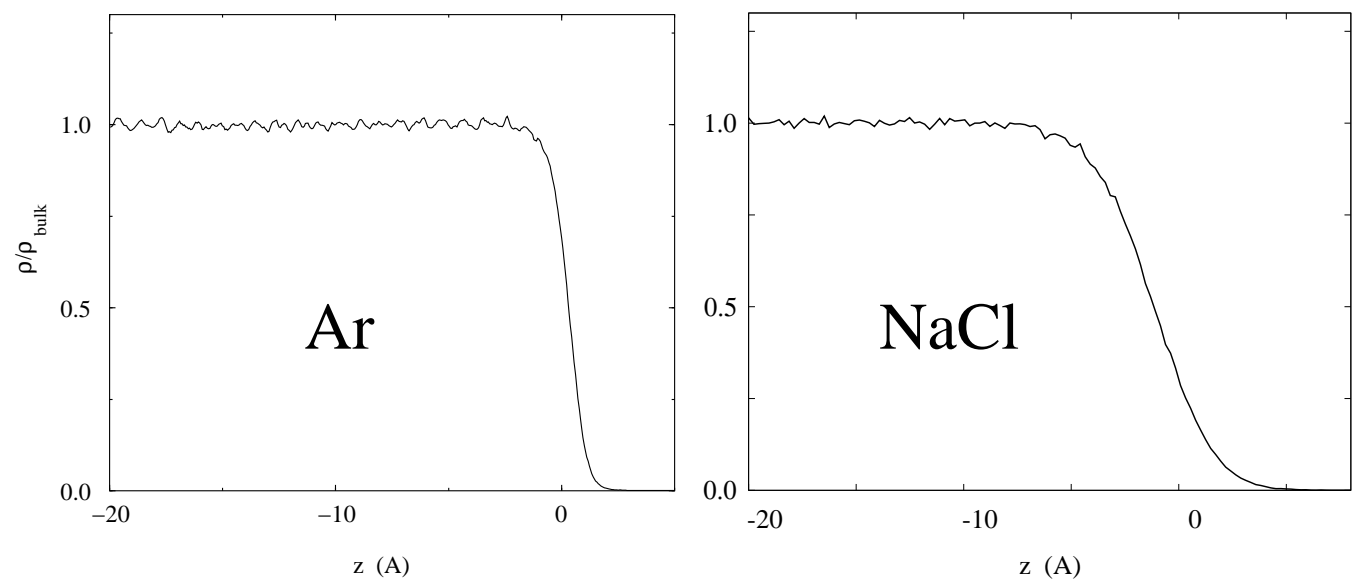

Fig. 21. Averaged density profile of the liquid surface of LJ (Ar) and NaCl obtained from a simulation at $T_{m}$. The density drops smoothly from the bulk value in the liquid phase to that of the vapor phase, and the layering oscillations are undetectable. 


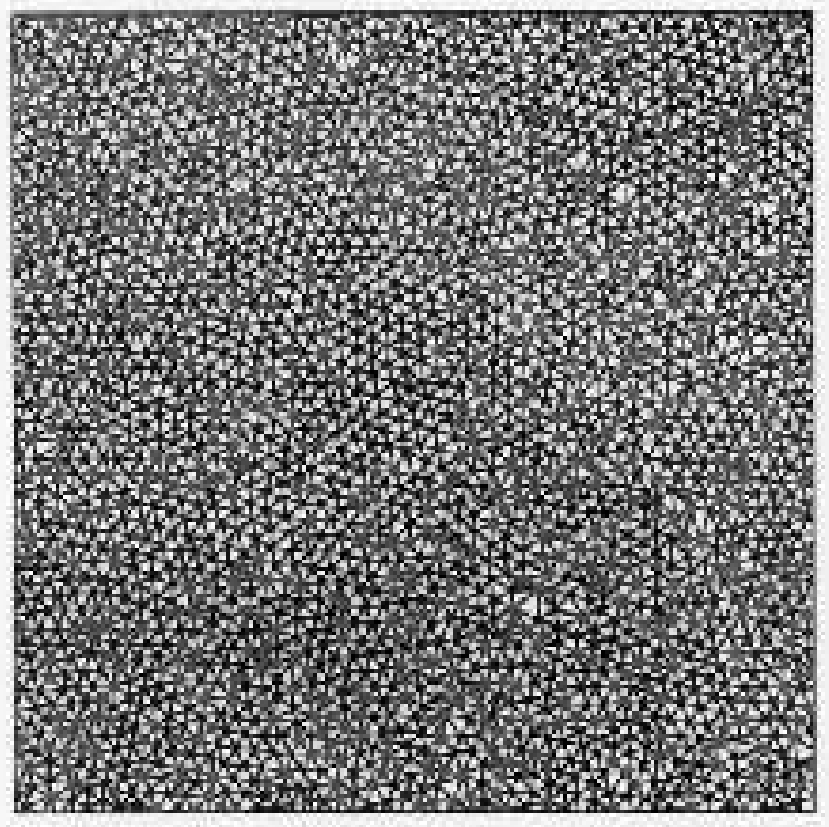

$$
\mathrm{T}=1450 \mathrm{~K}
$$

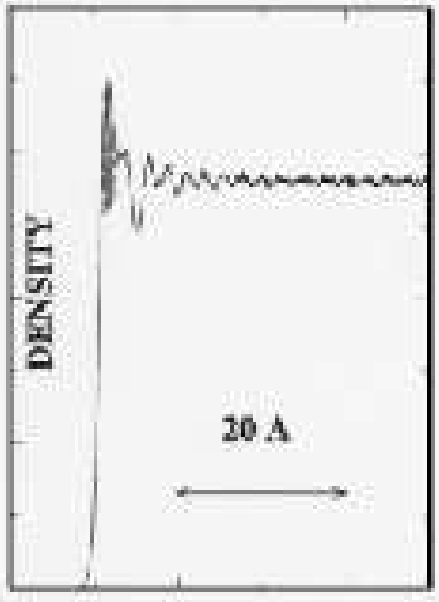

DEPTH

\section{$\mathbf{T}=1000 \mathrm{~K}$}
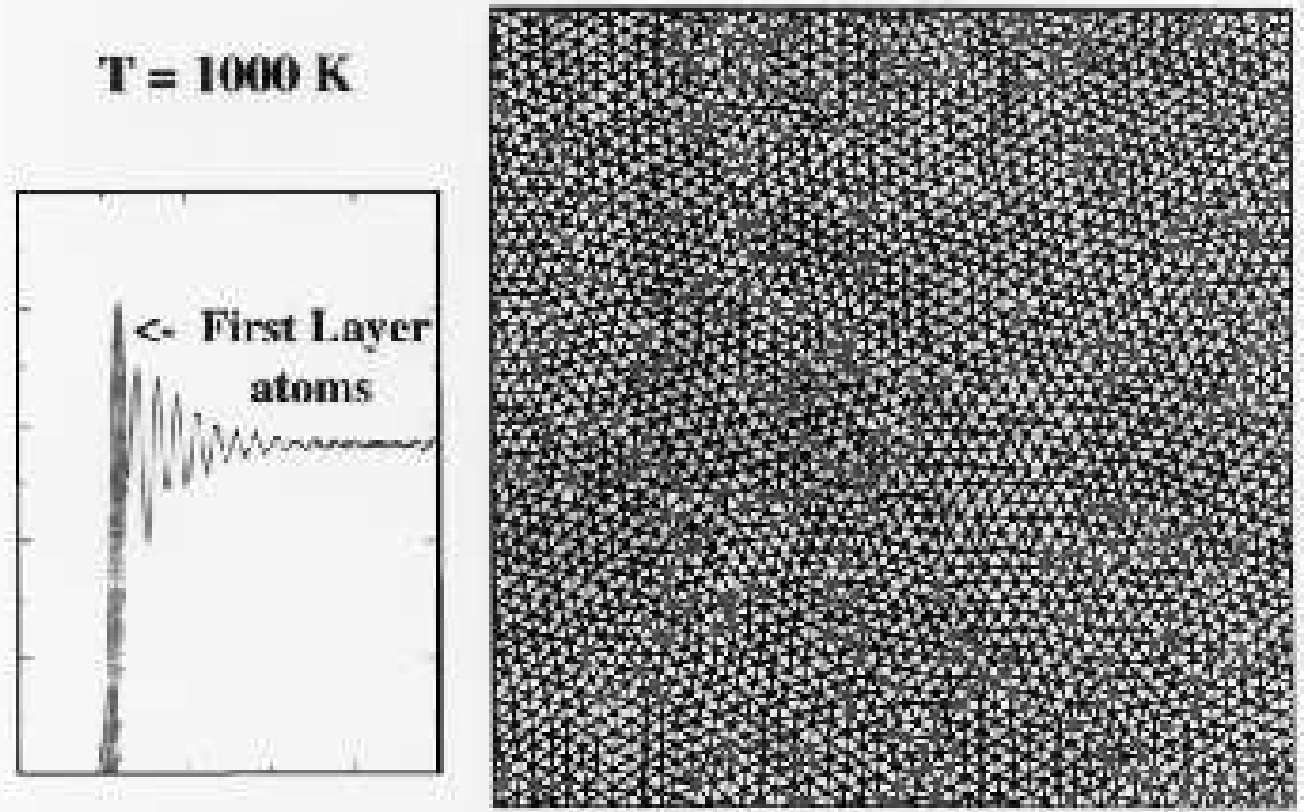

Fig. 22. Nearly hexatic top layer of simulated liquid Au both above and below (undercooling) the melting point $T_{m}=1335 \mathrm{~K}$. Fivefold (green) and sevenfold (red) disclinations are pinpointed. The corresponding density profiles along the surface normal are also shown. The first peak corresponds to the outmost layer of atoms in the maps. From Ref. [86]. 


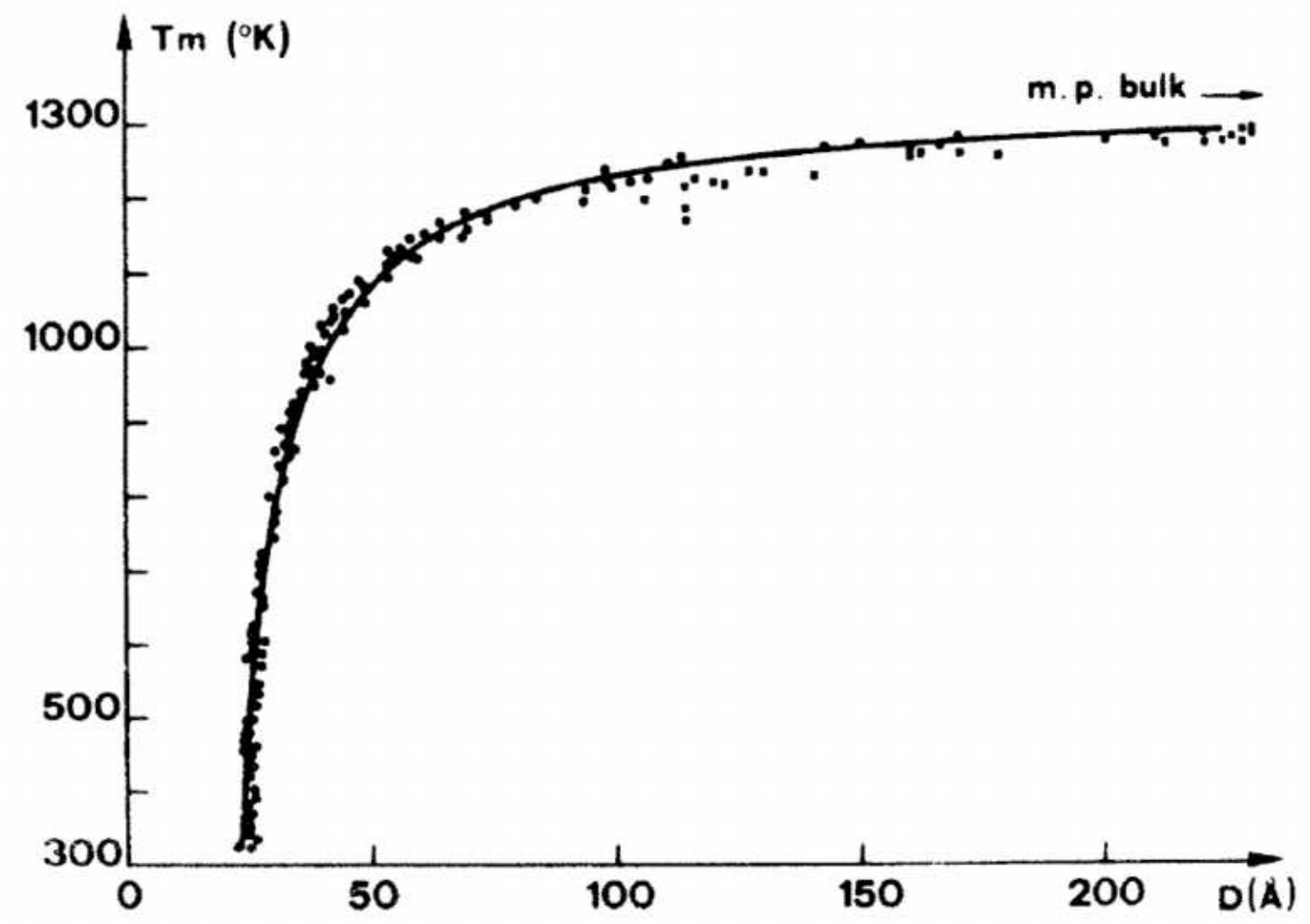

Fig. 23. Experimental values of the melting point temperature of gold particles. From Ref. [87]. 


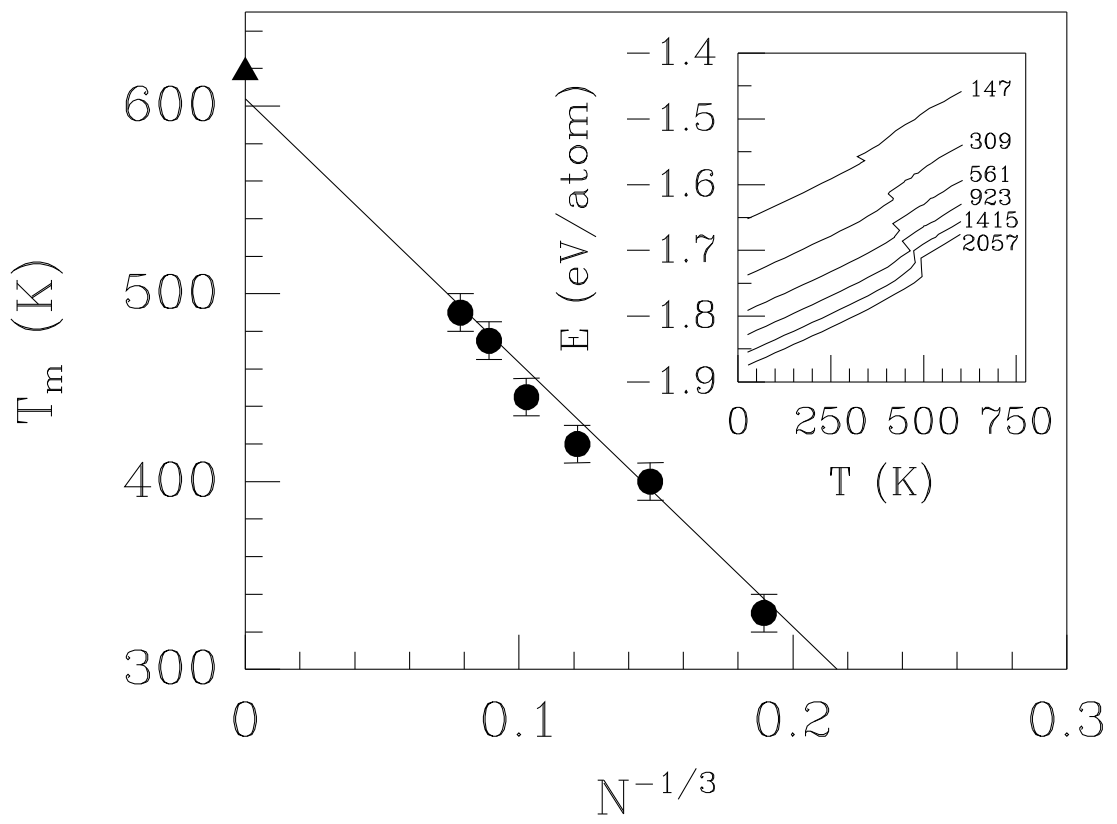

Fig. 24. Melting temperature as a function of $N^{-1 / 3}$ (where $N$ is the number of atoms) of simulated $\mathrm{Pb}$ clusters. The points correspond to the discontinuities in the caloric curves in the inset. The solid line is the linear fit to the points. The triangle corresponds to bulk melting.From Ref. [92] 


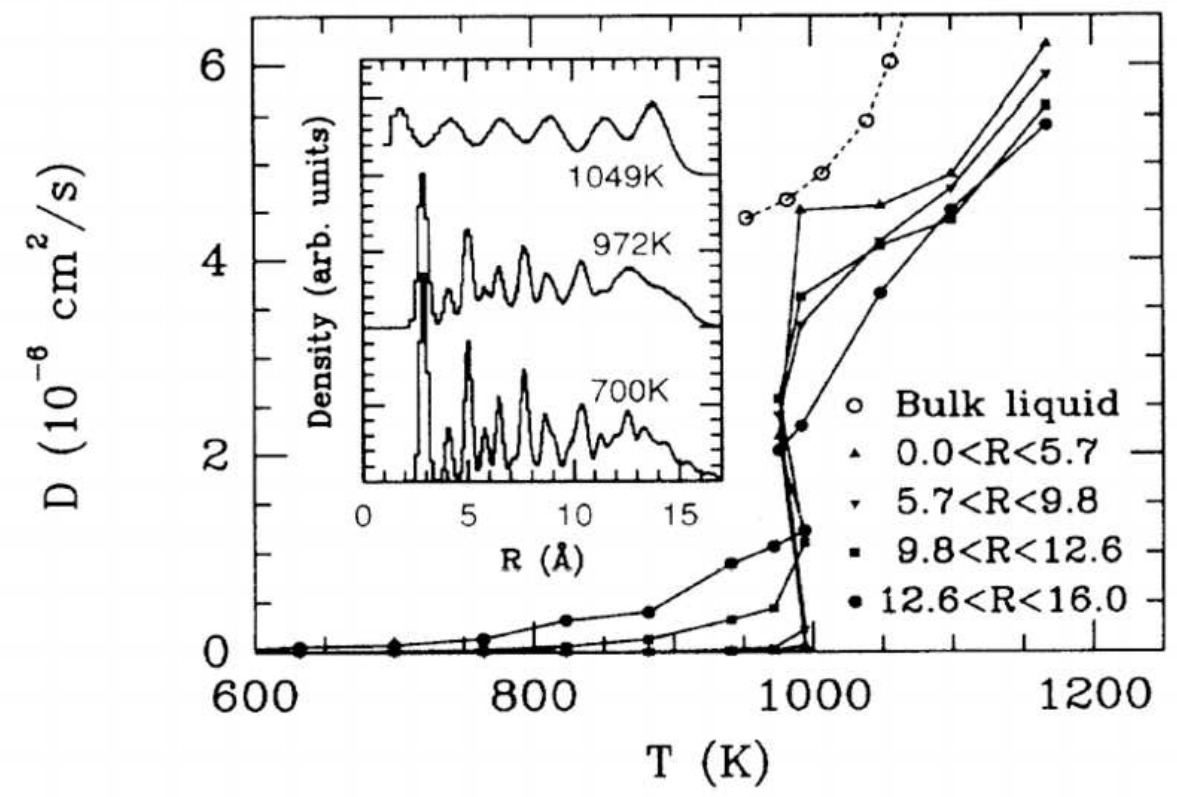

Fig. 25. $\mathrm{Au}_{879}$ : $T$ dependence of the diffusion coefficient for different shells, compared with that for the supercooled bulk liquid. Inset: Radial density distribution at $\mathrm{T}=700,972$ and $1049 \mathrm{~K}$. 

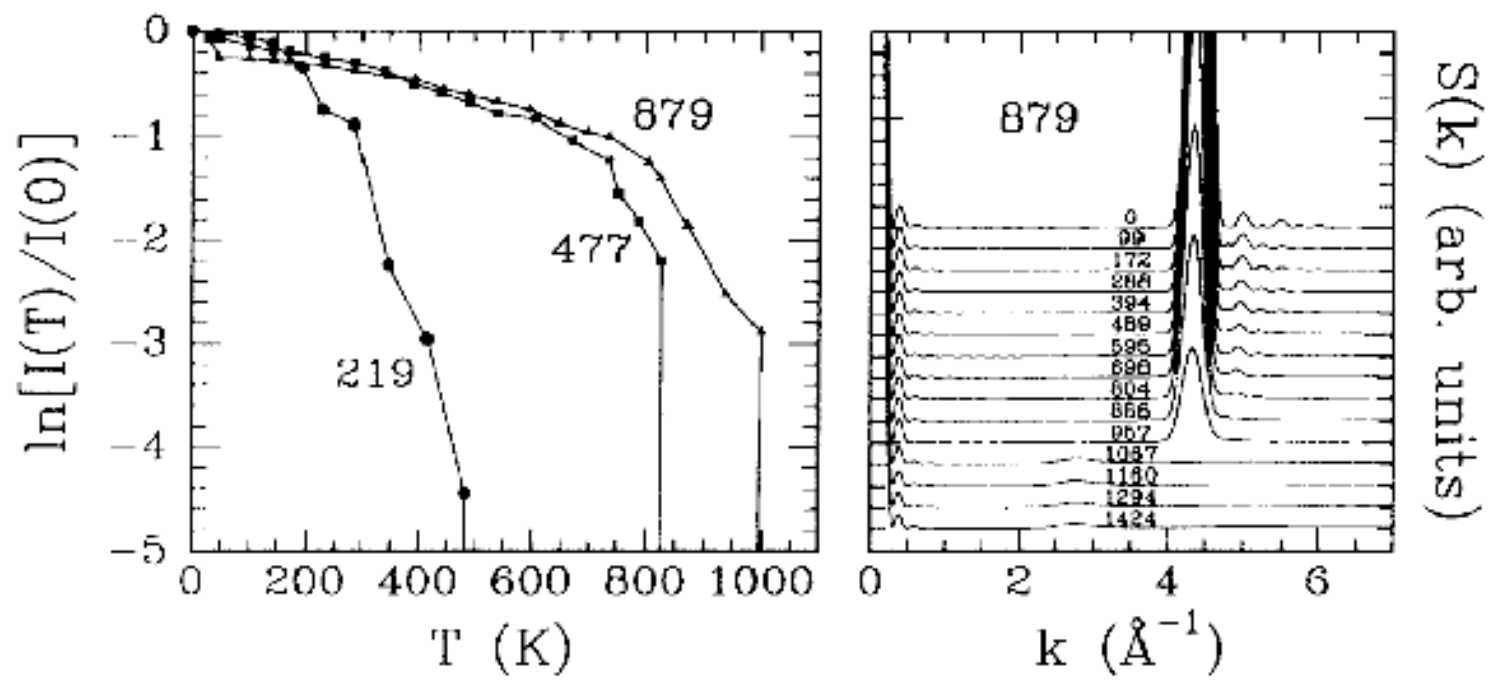

Fig. 26. Logarithm of the effective Debye-Waller factor as a function of $T$ for $\mathrm{Au}_{219}$, $\mathrm{Au}_{477}$, and $\mathrm{Au}_{879}$, and $T$ dependence of $S(k)$, with $k$ along the (110) direction, for $\mathrm{Au}_{879}$. From Ref. [93]. 